\title{
Overexpression of SMYD3 contributes to sorafenib resistance by epigenetically activating the expression of multiple cancer-promoting genes in hepatocellular carcinoma
}

\author{
Shanshan Wang \\ Hangzhou Red Cross Hospital https://orcid.org/0000-0001-7364-768X \\ Xinxin You \\ Xidian University \\ Fengwei Zhang \\ Hangzhou Red Cross Hospital \\ Hongjuan Zhou \\ Hangzhou Red Cross Hospital \\ Xuechai Shang \\ Hangzhou Red Cross Hospital \\ Cai Long ( $\sim$ c13588709359@163.com) \\ Zhejiang Province, People's Republic of China
}

\section{Research}

Keywords: Cancer stem cells, epithelial-mesenchymal transition, Sorafenib resistance, SMYD3, SMYD3mediated cancer-promoting genes

Posted Date: January 19th, 2021

DOl: https://doi.org/10.21203/rs.3.rs-147859/v1

License: (c) (i) This work is licensed under a Creative Commons Attribution 4.0 International License. Read Full License 


\section{Abstract \\ Background}

The resistance mechanism to sorafenib in hepatocellular carcinoma (HCC) remains poorly understood. Recent evidence has demonstrated the enrichment of liver cancer stem cells (CSCs) as culprit for treatment resistance. In liver cancer development, SMYD3 epigenetically activates or overexpresses JAK/STAT3 pathway, epithelial-mesenchymal transition (EMT) pathway, SOX4 and MYC oncogenes, which play crucial roles in liver CSC. In this study, we demonstrate the novel role of SMYD3 in HCC resistance to sorafenib therapy.

\section{Methods}

We used sorafenib-resistant HCC in vitro and in vivo models to study the relationship between sorafenib resistance and SMYD3 expression. Chromatin immunoprecipitation (ChIP) and quantitative real-time PCR (qRT-PCR) were used to analyze the mechanism of SMYD3 regulation. Stemness and metastatic properties were investigated after treatment with SMYD3 depletion alone or in combination with sorafenib to evaluate the therapeutic effect on sorafenib resistance by in vitro and in vivo experiments.

\section{Result}

We identified overexpression of SMYD3 and subsequent increase of histone H3K4me3 as a novel pathway of acquired resistance to sorafenib in HCC. We also found that multiple SMYD3-mediated cancer-promoting genes exhibited up-regulation in sorafenib-resistant HCC cells and tumors. Inhibition of SMYD3 by a small-molecular inhibitor BCL121 or genetic means suppressed the transcription of SMYD3 target genes via the inhibition of the recruitment of H3K4me3-midifed histone tails to the core promoter regions of these genes. Restoration of wide-type SMYD3 protein in sorafenib-resistant cells with SMYD3 knockdown partly rescued the expression of target genes, while mutant SMYD3 did not. As such, modulating SMYD3 expression or activity in vitro and in vivo models inhibited the transcription output of target genes, mainly through SOX4, MYC, JAK1 and ZEB1 genes, and suppressed activation of their associated pathways, including EMT, JAK/STAT3, SOX4 and MYC pathway, and consequently weakened the stemness and metastatic properties of sorafenib-resistant cells in vitro and suppressed the relapse and metastasis of sorafenib-resistant tumors in vivo.

\section{Conclusion}

SMYD3 conferred sorafenib-resistant cells enhanced stemness and metastatic properties in HCC by epigenetically activating the expression of multiple cancer-promoting genes. SMYD3 could be a rational target for therapeutic intervention in sorafenib-resistant HCC. 


\section{Background}

Sorafenib is a standard treatment for patients with advanced hepatocellular carcinoma (HCC) [1]. However, therapeutic resistance and relapse are common and represent major obstacles to the improvement of patient survival in HCC [2]. Evidence indicates that liver cancer stem cells (CSCs) accumulate after long-term sorafenib treatments and are likely to contribute to their failure and subsequent generation of metastasis and local recurrence [3-5]. The development of liver CSCs and maintenance of their "stemness" are associated with aberrations of several molecular cascades involving signaling triggered by Notch, Wnt/beta-catenin, JAK/STAT3, SOX4 and MYC, which are regulated and defined by genetic and epigenetic mechanisms [4,6-10]. Therefore, intervention of CSCs self-renewal by epigenetic regulators maybe a potential strategy for evading sorafenib resistance in HCC.

Epithelial mesenchymal transition (EMT) confers metastatic properties upon liver cancer cells by enhancing invasion,metastasis and resistance to drug treatment including sorafenib [3, 11-14]. EMT has been implicated in the generation of cancer cells with stem cell-like characteristics including increased self-renewal, tumor-initiating capabilities $[12,13,15]$. In addition, core EMT programs involving EMT transcription factors (SNAIL1, ZEB1, TWIST1, etc.) and epigenetic regulators accompany cell plasticity $[11,12,16-20]$. Therefore, blocking EMT pathways by epigenetic regulators constitutes an attractive strategy for sorafenib-resistant cancer treatment.

Aberrant Epigenetic regulators have been associated with liver CSCs, EMT, and chemoresistance including sorafenib in HCC [4, 21]. Alterations of epigenetic modifiers can determine the outcome of HCC $[4,21,22]$. What's more, the activity of established and investigational epigenetic therapies, including inhibitors of DNA methyltransferases (DNMT), histone deacetylases (HDAC), histone lysine methytransferases (KMTs) and histone lysine demethylases (HDMs), in well-defined clinical contexts has provided evidence that this strategy can be effective [22]. Therefore, achieving a deep understanding of epigenetic mechanisms is needed to develop better therapies.

SMYD3 is a member of the SMYD lysine methylase family and plays an important role in the methylation of various histone and non-histone targets [23, 24]. SMYD3 was descripted as a histone 3 lysine-4 (H3K4) methyltransferase, which displays oncogenic activity in liver cancer [24, 25]. It is always overexpressed in several tumors including HCC, and plays an important role in modulating several cellular processes involved in cell survival, oncogene activation and epithelial to mesenchymal transition (EMT) [24]. What's more, SMYD3 expression in human HCC has a prognostic value. SMYD3 expression correlated with the probability of overall survival, the probability of surviving as a "tumor-free" patient after chemotherapy, the probability of developing new tumor foci and the probability of progressing to high-grade (G3/G4) tumors for a given time frame in HCC [24]. However, direct demonstration about the role of SMYD3 in acquired resistance to sorafenib of $\mathrm{HCC}$ is lacking, and the responsible mechanisms need further investigation.

In liver cancer development, SMYD3, as a transcriptional potentiator of multiple cancer-promoting genes, including SNAIL 1, TWIST1, ZEB1, PCNA, CCNE1, CCNA2, MYC, JAK1, JAK2 and IGFPB1, directly interacts with H3K4me3-modified histone tails and is recruited to the core promoter regions of active genes [24]. 
SNAIL1, TWIST1 and ZEB1 are key EMT transcription factors [12, 15-17]. Overexpression of JAK1 and JAK2 promote the activation of JAK/STAT3 signaling pathway [24]. Activated JAK/STAT3 pathway or overexpressed SOX4 or MYC protein is required for liver CSCs self-renewal $[7,9,10]$. Besides, inhibiting them is beneficial to the elimination of cancer stem cells $[7,9,10]$. Given the critical role of these targets in liver cancer, SMYD3 maybe is a promising candidate to targeting liver CSCs and EMT.

Here, we found that SMYD3 is overexpressed in sorafenib-resistant in vivo and in vitro models, and overexpressed SMYD3 confers hepatoma cells sorafenib resistance through enhanced stem-cell and metastatic properties. Multiple cancer-promoting genes mediated by SMYD3 were up-regulated in Sorafenib-resistant cells and tumors. Furthermore, molecular and pharmacological inhibition of SMYD3 attenuated EMT, JAK/STAT3, SOX4 and MYC pathway by decreasing the expression of SMYD3-mediated cancer-promoting genes, mainly through ZEB1, JAK1, SOX4 and MYC gene, and consequently weakened self-renewal ability and migration and invasion ability in vitro, and then suppressed sorafenib-resistant cancer relapse and metastasis in vivo. Taken together, these results suggest that pharmacological targeting of SMYD3 is a promising strategy to overcome sorafenib resistance in HCC.

\section{Methods}

\section{Cell culture}

Human hepatoma cell lines Huh7, SMMC-7721, BEL-7404 and SK-Hep1 were obtained directly from Shanghai Cell Bank of Chinese Academy of Sciences (Shanghai, China) [26]. Cells were maintained in Dulbecco's modified eagle medium (DMEM) supplemented with 10\% fetal bovine serum (FBS) and 1\% penicillin-streptomycin. All cultures were maintained in a humidified incubator at $37^{\circ} \mathrm{C}$ and $5 \% \mathrm{CO}_{2}$. The cell lines have been characterized at the cell bank by DNA fingerprinting analysis using short tandem repeat markers. All cell lines were placed under cryostage after they were obtained from the cell bank and used within 6 months of thawing fresh vials, as described previously $[4,26]$.

\section{Construction of plasmids, shRNAs, transfection, and lentivirus-mediated gene knockdown}

For expression of wide type SMYD3, the cDNA was amplified by PCR and ligated into the correct reading frames of pcDNA3.0-flag vector containing FLAG coding sequences as described previously [26]. To generate catalytic dead mutant SMYD3-Y239F expression vectors, SMYD3 cDNA was mutated by the QuikChange® II site-directed mutagenesis kit (Agilent Technologies) before the construction [27]. All plasmid constructs were confirmed by DNA sequencing. Plasmid transfections were performed using Lipofectamine 2000 (Invitrogen, USA). The shRNA against SMYD3 gene shSMYD3s and corresponding nonspecific shRNA (Sigma, USA) were used for RNA interference as described previously $[4,26]$. The sequences are listed in Supplementary Table S1.

\section{Treatment of cells with inhibitors in vitro and establishment of sorafenib-resistant cells in vitro}


Different amounts of inhibitor(s) were dissolved in dimethyl sulfoxide. Cells were plated in 6-well plates. When cells reached $60 \%$ confluence, they were treated with the appropriate dose of inhibitor at different times. After treatment, cells were collected for further experiments and analyses. Chemical reagents are listed in Supplementary Table S2.

Sorafenib-resistant cells in vitro from Huh7, SMMC-7721, BEL-7404 and SK-Hep1 cells were established as described previously [4]. Briefly, Huh7, SMMC-7721, BEL-7404 and SK-Hep1 cells were exposed to sorafenib from low dose $(2.5 \mu \mathrm{M})$ and when cells stably grew then started to change to the higher dosage of sorafenib $(5,7.5$, and then $10 \mu \mathrm{M})$. Medium containing sorafenib was replaced every two days for three months. In the ends, the cells could grow slowly in medium containing $10 \mu \mathrm{M}$ sorafenib (a clinically relevant dose).

\section{Western blotting and immunofluorescence assay}

Proteins extracted for western blotting assay were resolved by sodium dodecyl sulfate-polyacrylamide gel electrophoresis (SDS-PAGE), transferred to polyvinylidene fluoride membranes, and blotted with primary and secondary antibodies. Densitometry was performed using ImageJ $1.51 \mathrm{~h} v$ software (http://rsb.info.nih.gov/ij/). Regarding the immunofluorescence assay, cells were fixed, and then blocked with $1 \%$ bovine serum albumin-phosphate-buffered saline prior to incubation with primary and secondary antibodies. Antibody list used is shown in Supplementary Table S2.

\section{RNA extraction and quantitative real-time PCR (qRT-PCR)}

Total RNA was isolated using TRIzol reagent (Invitrogen, NY) and reverse transcription was performed with 500ng of RNA using PrimeScript ${ }^{\text {TM }}$ RT Master Mix (Takara, Japan), according to the manufacturer's instructions. qPCR was performed with FastStart Universal SYBR ${ }^{\circledR}$ Green Master (Roche, USA) on an Applied Biosystems 7500 Real Time PCR System supplied with analytical software (Applied Biosystems, USA). The average of the technical replicated was normalized to GAPDH levels using the comparative CT method $\left(2^{-\triangle \triangle C T}\right)$. Average and standard deviations of at least 3 experiments are shown in the figures. The primer pairs were listed in Supplementary Table S3.

\section{Annexin V / Propidium iodide staining and flow cytometry analysis}

Annexin $\mathrm{V}$ / propidium iodide $\mathrm{PI}$ ) staining and flow cytometry analysis were carried out using the Annexin V-FITC Apoptosis Detection Kit (BD Biosciences, USA) according to the manufacturers' instructions.

\section{Colony formation assay}

Two days after transfection, $310^{3}$ tumor cells per well were resuspended in DMEM containing $0.3 \%$ agarose (Promega, USA). This suspension was laid over DMEM containing $0.6 \%$ noble agarose in 6-well plates and further overlaid with DMEM. The plates were then incubated for 14 days in a $5 \% \mathrm{CO}_{2}$ incubator at $37^{\circ} \mathrm{C}$, with replenishment of medium every other day. Colonies were imaged using Olympus IX71 and macroscopically visible colonies in three randomly chosen fields per well were counted for quantification. 


\section{Sphere formation assay}

Spheres were generated in DMEM:F12 (Life Technologies, , USA) supplemented with 2\% B-27 (Life Technologies, USA), EGF, bFGF (PeproTech, USA), $100 \mathrm{IU} / \mathrm{mL}$ penicillin, and $100 \mathrm{mg} / \mathrm{mL}$ streptomycin on ultralow attachment plates for 14 days. Spheres were imaged using Olympus IX71 and macroscopically visible colonies in three randomly chosen fields per well were counted for quantification.

\section{Chromatin immunoprecipitation (ChIP)}

Chromatin immunoprecipitation (ChIP) was carried out with the ChIP Assay Kit (Millipore, USA) according to the manufacturer's instructions as described previously $[4,26]$. The percentage of the bound DNA was quantified against the original DNA input by PCR analysis. The PCR primer sets used are shown in Supplementary Table S1.

\section{Migration and invasion assays}

Migration and invasion were assessed using transwell assays with polycarbonate membrane inserts with 8.0 $\mathrm{MM}$ pore size (Corning) coated with or without Matrigel (Corning) as described previously [28].

\section{Animal models, histology, immunohistochemistry analysis, and evaluation}

All animal experiments were approved by the Institutional Animal Care and Use Committee of Zhejiang Chinese Medical University (Zhejiang, China) and carried out in accordance with the approved guideline "code of practice: animal experiments in cancer research" (Netherlands Inspectorate for Health Protection, Commodities and Veterinary Public Health, 1999).

Sorafenib-resistant subcutaneous tumors were generated as described previously [4]. Briefly, $1 \times 10^{7} \mathrm{Huh} 7$ cells in $200 \mu$ phosphate buffered saline were injected subcutaneously in 3-4 week-old male nude mice. After two weeks, mice were randomly allocated into groups and treated with sorafenib (60 $\mathrm{mg} / \mathrm{kg} /$ intraperitoneal injection (i.p.), once every other day) for two months. On day 60 after the start of treatment, tumors were removed.

To establish in vivo tumor formation model, 100 to 10,000 Huh7-Res or 7721-Res cells with NS shRNA or shSMYD3-\#1, were mixed with Matrigel, and were injected subcutaneously into the flanks of three-four week-old male nude mice. Tumors grew for approximately 4 weeks and were removed.

$1 \times 10^{7}$ Huh7-Res or Huh7-Res shSMYD3-\#1 cells in $200 \mu$ phosphate buffered saline were injected subcutaneously in 3-4 week-old male nude mice. After two weeks, mice with Huh7-Res cells were randomly allocated into 4 groups and treated with DMSO, sorafenib $(60 \mathrm{mg} / \mathrm{kg} /$ intraperitoneal injection (i.p.)), BCL121 (5 mg/kg/ i.p.), sorafenib (60 mg/kg/i.p.) plus BCL121 (5 mg/kg/i.p.)) for 4 weeks. Mice with Huh7-Res shSMYD3-\#1 cells were randomly allocated into 2 groups and treated with DMSO or sorafenib (60 mg/kg/ i.p.) for 4 weeks. 
Tumors were measured twice weekly and volumes. Tumor size was measured with digital calipers and calculated based on the following formula: length(width) ${ }^{2} / 6$. Tumors sections from subcutaneous tumor xenografted male nude mice were H\&E stained, immunohistochemically analyzed, and evaluated as described previously [4].

\section{Statistical analysis}

Experimental data were presented as mean \pm standard error of mean of at least 3 independent replicates through analyzing with GraphPad Prism 5 (GraphPad Software, La Jolla, CA) and assessing comparisons between different groups by the Student $t$ test, one-way ANOVA, and two-way ANOVA analysis of variance.

\section{Results}

\section{SMYD3 is overexpressed in sorafenib-resistant tumors and cells.}

Establishment of different sorafenib-resistant HCC cell lines has previously been successful in vitro and in vivo [4]. These cells were found to exhibit enhanced anti-apoptosis ability, slow cell-cycle and liver CSC properties, including enhanced tumorigenecity, self-renewal ability, and migration and invasion ability in vitro, by Annexin V staining, IP staining, colony and sphere formation assay, and migration and invasion assay (Fig. 1a) [4]. These cells exhibited enhanced metastatic potential, including liver and lung metastasis, upon long-term exposure to sorafenib in vivo (Fig. 1b) [4]. These results are in line with the finding that label-retaining liver cancer cells with resistance to sorafenib [5].

In order to study the role of SMYD3 in the treatment of sorafenib resistance in liver cancer, we firstly detected the expression of SMYD3 in sorafenib-resistant cell lines. Western blotting revealed that SMYD3 protein was highly expressed in sorafenib-resistant cell lines (Fig. 1C). Immunofluorescence assay further confirmed that the expression of SMYD3 in Huh7-derived sorafenib-resistant cells was significantly increased (Fig. 1C). Although it was also expressed in the nucleus, it was mainly enriched in the cytoplasm (Fig. 1c). The quantitative RT-PCR (qRT-PCR) method further confirmed the expression of SMYD3 at the transcription level was also significantly increased (Fig. 1c). Next, the SMYD3 protein level was detected by western blotting and immunohistochemistry in sorafenib-resistant tumors, and it was found that compared with control tumors, the SMYD3 protein level in sorafenib-resistant tumors was significantly increased (Fig. 1d). And qRT-PCR method further confirmed that the mRNA level of SMYD3 in sorafenib-resistant tissues was also significantly increased (Fig. 1d). Finally, we analyzed the liver cancer samples in the TCGA database and selected 3 groups: the group that did not receive chemotherapy drugs, the group that received sorafenib or other drug treatment groups, and compared the sequencing results of SMYD3 to find that compared with the untreated group, the expression of SMYD3 from drug-treatment groups was significantly higher, but there was no significant difference between sorafenib and other treatment groups (Fig. 1e). Therefore, SMYD3 is overexpressed in sorafenib-resistant cells and tumors. 


\section{Sorafenib-resistant cells exhibit widespread up-regulation of multiple cancer-promoting genes mediated by SMYD3.}

In the progression of liver cancer, SMYD3 invades active chromatin domains via association with H3K4Me3 and RNA Pol-II, thereby stimulating the transcription of key genes in cancer-related pathways [24]. These genes include: JAK1, JAK2, SNAIL 1, TWIST1, ZEB1, SOX4, MYC, IGFBP1, PCNA, CCNE1 and CCNA2 [24]. Therefore, as a first step toward understanding the significance of targeted SMYD3 therapy, we measured the expression of these genes in sorafenib-resistant cells and tumors by qRT-PCR. We found that a large majority of sorafenib-resistant samples exhibited a significant up-regulation the expression of these oncogenes compared with control samples (Fig. 2a). For example, except for IGFBP1 gene in Huh7Res, JAK2, SOX4, SNAIL 1, CCNE1, IGFBP1, and PCNA gene in 7404-Res cells, TWIST1 gene in 7721-Res cells, CCNA2 in SK-Hep1-Res cells, all other resistant cells expressed significantly higher levels of JAK1, JAK2, SNAIL 1, TWIST1, ZEB1, SOX4, and MYC gene compared with parental cells (Fig. 2a). In sorafenibresistant tumors derived from Huh7 cells, JAK1, SNAIL 1, TWIST1, ZEB1, SOX4, and MYC gene were significantly higher compared with DMSO-treated tumors (Fig. 2b). However, JAK2, SOX4, IGFBP1, PCNA, $C C N E 1$, and $C C N A 2$ gene were not significantly up-regulated in sorafenib-resistant tumors, but some tended to be up-regulated (Fig. 2b). Therefore, sorafenib-resistant cells exhibit widespread up-regulation of multiple cancer-promoting genes in vitro and in vivo.

\section{Genetic and pharmacological inhibition of SMYD3 suppresses the expression of multiple cancer- promoting genes and activation of their associated signaling pathways in sorafenib-resistant cells.}

Next, we investigate whether SMYD3 regulates the expression of these cancer-promoting genes. We conducted loss-of-function analysis of SMYD3 in vitro, and achieved the stable knockdown of SMYD3 in Huh7-Res and 7721-Res cells with lentivirus-mediated-shRNA against SMYD3 (shSMYD3). Two different shSMYD3, labeled shSMYD3-\#1 and shSMYD3-\#2, effectively suppressed the expression of SMYD3 protein and mRNA levels (Fig. 3a and b). After silencing SMYD3 or inhibiting the activity of SMYD3 with a small molecule inhibitor BCL121 [29], qRT-PCR was used to detect the effect on the transcription level of these cancer-promoting genes, and it was found that both significantly reduced the expression of their mRNA (Fig. 3a and c). Western blotting assay was used to detect the effect on their proteins and found that SMYD3 depletion significantly reduced the protein contents of these genes in sorafenib-resistant cell lines, thereby inhibiting the phosphorylation of STAT3 and N-cadherin protein, as well as the increase of E-cadherin protein, suggesting that SMYD3 depletion inhibited cell stemness, activation of JAK/STAT3 and EMT pathway (Fig. 3b and d). However, the synergy of the combined use of BCL121 and sorafenib is not significant in the transcriptional level, but it is significant in the protein level (Fig. $3 \mathrm{c}$ and d). Therefore, these findings indicated that SMYD3 depletion inhibited the expression of these oncogenes and the activation of related signal pathways. Moreover, BCL121 also played a similar inhibitory effect on the expression of SMYD3 target genes, indicating that this effect requires the participation of SMYD3 enzyme activity. 
SMYD3 epigenetically activated the expression of multiple cancer-promoting genes and is dependent on its HMT activity.

In order to further confirm the role of SMYD3 in the regulation of these genes, we conducted gain-offunction analysis of SMYD3 in vitro in sorafenib-resistant cells with SMYD3 knockdown. We constructed wild-type SMYD3 (SMYD3) and catalytic dead mutant SMYD3-Y239F (SMYD3-M) in the pcDNA3.1-flag vector, and transferred wild-type SMYD3 or mutant SMYD3 to sorafenib-resistant cells with SMYD3 knockdown, and studied the effect on the transcription level of target genes. Because knockdown of SMYD3 was more prominent with shSMYD3-\#1 than shSMYD3-\#2, we used shSMYD3-\#1 for most of subsequent experiments. Quantitative RT-PCR showed that the expression of wild-type SMYD3 complemented down-regulated the expression of multiple cancer-promoting factors caused by SMYD3 silencing, but not mutant SMYD3 (Fig. 4a). This result suggested that SMYD3 was necessary for the regulation of multiple cancer-promoting genes and dependent on its enzyme activity. In order to further evaluate the mechanism of SMYD3 in the regulation of these genes, chromotin immunoprecipitation (ChIP) assay was performed to detect the recruitment of SMYD3 and RNA polymerase II in the promoter regions of these oncogenes and the modification of histone H3K4me3 in sorafenib-resistant cells with SMYD3 silence or inhibition. JAK1, SOX4, and ZEB1 gene were selected as representatives for further research. It was found that the recruitment of SMYD3 and RNA polymerase II in the promoter regions of JAK1, SOX4, and ZEB1 gene was reduced, and histone H3K4Me3 modification was also significantly reduced by SMYD3 depletion (Fig. 4b and c). Secondly, we transferred wild-type SMYD3 and enzymemutated SMYD3 into sorafenib-resistant cells with SMYD3 knockdown, and performed ChIP assay with flag antibody, and found that both wild-type and mutant SMYD3 can bind to the promoter regions of JAK1, SOX4, and ZEB1 gene (Fig. 4d). However, compared with the mutant SMYD3, the recruitment of RNA polymerase II and histone $\mathrm{H} 3 \mathrm{~K} 4 \mathrm{me} 3$ modification on the detected promoters were significantly increased in cells expressing wild-type SMYD3 (Fig. 4d). These results showed that SMYD3 epigenetically regulated the expression of these cancer-promoting genes via histone H3K4me3 modification.

\section{Genetic and pharmacological inhibition of SMYD3 suppresses tumorigenecity, self-renewal ability, and migration and invasion ability in HMT-dependent manner.}

Drug-resistant cells have more cancer stem cells (CSCs), resulting in cancer relapse and metastasis. SMYD3-mediated cancer-promoting genes and their associated pathway directly or indirectly involved in liver CSCs. After confirming that both genetic and pharmacological inhibition of SMYD3 resulted in suppressing JAK/STAT3, SOX4, MYC, and EMT pathway, we tested if the treatment of sorafenib-resistant HCC cells with SMYD3 knockdown or inhibition result in decreasing these pathways mediated tumorigenecity, self-renewal, and migration and invasion ability. Regarding the relative self-renewal potential of sorafenib-resistant cells, silencing SMYD3 significantly decreased colony and sphere formation frequencies than controls (Fig. 5a). Consistent with this finding, limiting dilution analysis showed that sorafenib-resistant cells with shSMYD3 required more cells and longer incubation times to generate tumors in vivo as compared those with nonspecific shRNA, indicating that sorafenib-resistant cells with shSMYD3 were significantly less enriched in CSCs (Fig. 5b). To confirm the results obtained 
using SMYD3 shRNA, we treated sorafenib-resistant cells with SMYD3 inhibitor BCL121 and measured tumorigenecity and self-renewal ability. In agreement with the results observed with SMYD3 knockdown, SMYD3 inhibitor also resulted in decreasing colony and sphere formation frequencies, and the combination with sorafenib also has a significant synergistic effect (Fig. 5c). We further recovered SMYD3 expression to rescue the weakening of the stemness of sorafnieb-resistant cells caused by SMYD3 knockdown, and found that overexpression of wide-type SMYD3 significantly restored tumorigenecity and self-renewal ability of sorafenib-resistant cells, but not mutant SMYD3(Fig. 5d). Regarding the relative migration and invasion ability of sorafenib-resistant cells, we found that SMYD3 knockdown or SMYD3 inhibition by BCL121 significantly inhibited migration and invasion ability of sorafenib-resistant cells using transwell assay with or without matrigel (Fig. 6a-d). What's more, overexpression of wild-type SMYD3 significantly restored the migration and invasion capabilities of sorafenib-resistant cells upon SMYD3 knockdown, but not mutant SMYD3 (Fig. 6e-f). These findings indicated that SMYD3 depletion could effectively inhibit the stemness, migration and invasion ability of sorafenib-resistant cells in HMT-dependent manner.

\section{Genetic and pharmacological inhibition of SMYD3 inhibited the growth and metastasis of sorafenib- resistant tumors by inhibiting the expression of SMYD3-related cancer-promoting genes in vivo.}

To evaluate to the impact of targeting SMYD3 in the treatment of sorafenib-resistant tumors, we used a xenograft model to test the effects of shSMYD3 or BCL121 on sorafenib-resistant tumor growth and metastasis. We subcutaneously injected Huh7-Res cells or Huh7-Res with stably expressing shSMYD3-\#1 vector, and treated with sorafenib or not, or treated with BCL121 or not, and divided into 6 groups, including DMSO group, Sorafenib group, shSMYD3-\#1 DMSO group, shSMYD3-\#1 sorafenib group, BCL121 group and Sorafenib + BCL121 group (Fig. 7a). First, we found that compared with the DMSO treatment, sorafenib treatment continued to inhibit the growth of sorafenib-resistant tumors in vivo (Fig. 7b-c). Second, targeting SMYD3 by shSMYD3 or BCL121 both significantly inhibited the growth of sorafenib-resistant tumors, and silencing SMYD3 combined with sorafenib had a good synergistic effect on tumor growth, while BCL121 combined with sorafenib had not (Fig. 7b-c). Because Sorafenib-resistant cells acquire stem cell properties and metastatic properties, which induce cancer relapse and metastasis in multiple organs of nude mice [4,5]. Finally, we studied the metastasis in liver, lung and spleen by H\&E staining, and found that the DMSO and sorafenib treatment groups developed multiple obvious liver and lung metastases, with abnormal inflammatory cell enrichment in the spleen, while SMYD3 depletion by shSMYD3 or BCL121 had no obvious liver or lung metastasis was found in the group and its combination group with sorafenib, but there was still abnormal inflammatory cell enrichment in the lung and spleen (Fig. 7d). These findings directly show that SMYD3 depletion can effectively inhibit sorafenib-resistant tumor growth and distant metastasis in vivo.

To further confirm whether SMYD3 exerts a therapeutic effect by regulating the expression of related cancer-promoting genes in vivo, as a first step, we used qRT-PCR assay to detect the expression of SMYD3-related cancer-promoting genes at the transcriptional level (Fig. 8a). We firstly found that compared with the DMSO group, SMYD3 expression and its related multiple oncogene expressions were 
significantly increased in the sorafenib group (Fig. 8a). Secondly, compared with the sorafenib group, the expression of SMYD3 gene and its related multiple oncogenes, including SOX4, MYC, JAK1, ZEB1, and TWIST1 gene, were significantly reduced by SMYD3 depletion via shSMYD3 or BCL121, while their combination with sorafenib did not have a significant synergistic effect on these genes (Fig. 8a). In addition, for other genes such as CCNA2, CCNE1, and SNAIL 1 genes, silencing SMYD3 significantly inhibited the expression, while BCL121 treatment caused a slight decrease in expression but not significant (Fig. 8a). However, regardless of silencing SMYD3 or BCL121 treatment, the JAK2 gene expression decreased slightly, but there was no statistical significance. What's more, any treatment has no significant effect on the expression of PCNA gene (Fig. 8a). Next, we performed western blotting assay to detect the protein content of SMYD3-related cancer-promoting genes and related signaling pathways (Fig. 8b-c). The expression of five genes is consistent with qRT-PCR results, including SMYD3, SOX4, MYC, $J A K 1$, and ZEB1 gene, which was increased significantly in sorafenib group compared with DMSO group, and was partially reversed by SMYD3 depletion via shSMYD3 or BCL121, but was not synergistically affect by combination with sorafenib (Fig. 8b-c). Besides, another cell stemness marker NANOG protein, and activated marker of JAK/STAT signaling pathway phosphorylated STAT3 (pSTAT3) were similar trends with them (Fig. 8b-c). However, only silencing SMYD3 exhibited molecular alterations as demonstrated by the decreased expression of epithelial marker E-cadherin and increased expression of mesenchymal markers $\mathrm{N}$-cadherin, indicating that cancer cells was undergoing EMT. These findings showed that targeting SMYD3 inhibited the expression of multiple cancer-promoting genes, resulting in a significant reduction in protein production, thereby inhibiting the activation of corresponding signaling pathways such as stemness-related factors, JAK/STAT3 and EMT pathway.

\section{Discussion}

Previous studies have shown that SMYD3 expression correlates with tumor progression and poor prognosis of HCC [24]. SMYD3 has been identified as an attractive therapeutic target for liver cancer [24]. Because SMYD3, as an oncogene, functions in the nucleus, stimulating the transcription of several key regulators involved in cell proliferation, EMT, the JAK/STAT3 pathway, as well as the MYC and SOX4 oncogenes [24]. In this paper, we explored the role and mechanism of SMYD3 in sorafenib resistance and evaluated the therapeutic effect of inhibition of SMYD3 in sorafenib-resistant in vitro and in vivo models to improve sorafenib treatment efficacy. We firstly found that SMYD3 expression was consistently upregulated in all four established sorafenib-resistant HCC cell lines and sorafenib-resistant HCC tumors derived from Huh7 cells. In the TCGA database, compared with the untreated group, the expression of SMYD3 from drug-treatment groups was significantly higher, but there was no significant difference between sorafenib and other treatment groups. No matter it is due to the high expression of SMYD3 caused by drug treatment or the high malignancy of high-expressing SMYD3 tumors requiring targeted therapy or other chemotherapy, it can be determined that the SMYD3 mRNA level in the sorafenib treatment group is indeed higher. These results suggested the therapeutic potential of targeting SMYD3, as an oncogene, alone or together with sorafenib treatment in HCC. 
In term of mechanism, alterations of epigenetic modifiers can affect $\mathrm{HCC}$ epigenome in multiple aspects and determine HCC outcome [21,22, 24]. In chemically induced liver cancer development in mice, SMYD3 can directly interact with H3K4me3-modified histone tail, which facilitates its recruitment to active gene regulatory regions [24]. While in most locations the function of SMYD3 is neutral, on a specific set of genes SMYD3 acts as an essential transcriptional potentiator [24]. Except for promoting carcinogenesis, SMYD3 target genes are also key regulators involved in liver CSCs and EMT core programs $[7,9,10,12$, $15-17,30,31]$. Therefore, we next examine the expression of these specific genes mediated by SMYD3. As we expected, they exhibited widespread overexpression in all four established sorafenib-resistant HCC cell lines and sorafenib-resistant HCC tumors derived from Huh7 cells. SMYD3 was recruited to their transcriptional active region of the chromosome, coupling with histone H3K4Me3 modification and RNA polymerase II. SMYD3 depletion epigenetically decreased the expression of these genes via H3K4me3 modification, and indirectly or directly suppressed the activation JAK/STAT3, SOX4, MYC, and EMT pathway in sorafenib-resistant cells. Conversely, SMYD3 overexpression in sorafenib-resistant cells epigenetically activated the expression of multiple oncogenes, which in turn directly or indirectly activating JAK/STAT3, SOX4, MYC, and EMT pathway. The results present in this paper demonstrate that SMYD3 has a nuclear function, which is essential for the transcriptional regulation of multiple cancerpromoting genes in sorafenib-resistant cells.

To examine the role of SMYD3 in sorafenib resistance, we stably suppressed SMYD3 expression in two sorafenib-resistant cell lines highly expressing SMYD3 using a lentivirus-based approach, or treated two sorafenib-resistant cells with an inhibitor of SMYD3 activity BCL121. The results revealed that SMYD3 depletion alone or together with sorafenib both weakened tumorigenecity, self-renewal ability, and migration and invasion ability of sorafenib-resistant cells in vitro, and suppressed sorafenib-resistant tumors relapse and metastasis in vivo. What's more, the restoration of wide-type SMYD3 protein rescued weakened tumorigenecity, self-renewal ability, and migration and invasion ability of sorafenib-resistant cells upon SMYD3 knockdown, while the overexpression of HMT-mutant SMYD3 did not. These results suggested that SMYD3 played a functional role in enhanced liver CSC properties of sorafenib resistant cells in a HMT-dependent manner. However, like other solid cancers, other mechanisms modified by SMYD3 may also be involved in controlling acquired sorafenib resistance in HCC, for example, protein methylation-induced kinase group reprogramming and immune escape of cancer cells [32-34]. Therefore, we identified SMYD3 served as an important molecular switch controlling acquired sorafenib resistance in HCC.

\section{Conclusion}

In conclusion, our results indicated that sorafenib resistance in $\mathrm{HCC}$ was due in part to a compromised direct anti-tumor effect which appears to be regulated by SMYD3. Active multiple oncogene signalings by resistance to sorafenib were associated with epigenetic regulator SMYD3, which up-regulated the expression of multiple oncogenes as a mechanism to escape sorafenib stress. We anticipate that our findings will have direct clinical implications as SMYD3 inhibitors are already in advanced clinical development to overcome/delay HCC resistance. 


\section{Abbreviations}

CSCs: cancer stem cells; EMT: epithelial-mesenchymal transition; ChIP: Chromatin immunoprecipitation; qRT-PCR: quantitative real-time PCR; HCC: hepatocellular carcinoma; DNMT: DNA methyltransferases; HDAC: histone deacetylases; HDMs: histone lysine demethylases; FBS: fetal bovine serum; DMEM: Dulbecco's modified eagle medium; SDS-PAGE: sodium dodecyl sulfate-polyacrylamide gel electrophoresis; PI: propidium iodide; TCGA: The Cancer Genome Atlas; pSTAT3: phosphorylated STAT3

\section{Declarations}

\section{Ethics approval and consent to participate}

All animal work and procedures were approved by the Institutional Animal Care and Use Committee of Zhejiang Chinese Medical University (Zhejiang, China).

\section{Consent for publication}

Not applicable.

\section{Availability of data and materials}

The datasets generated/ analyzed during the current study are available

\section{Competing interests}

The authors declare that they have no competing interests.

\section{Funding}

This work was supported by grants from National Natural Science Fund of China (Grant Numbers 81402470), Zhejiang Basic Public Welfare Research Project (Grant Numbers Y21H100002), Medical Science and Technology Project of Zhejiang Province (Grant Numbers 2017KY128), Hangzhou Medical Science and Technology key Project (Grant Numbers 2014Z11), Hangzhou municipal autonomous application project of social development and scientific research (Grant Numbers, 20191203B134), and Hangzhou Red Cross Hospital project (Grant Numbers hhyn201301).

\section{Authors' contributions}

SSW and LC conceived the study and designed the major experiments. SSW, XXY, FWZ, HJZ and XCS performed experiments. SSW contributed to Materials and methods. SSW, FWZ, HJZ and XCS analyzed data. SSW wrote the manuscript. All authors read and approved the final manuscript.

\section{Acknowledgements}

No 


\section{Author details}

${ }^{1}$ Central Laboratory, Hangzhou Red Cross Hospital (Zhejiang Chinese Medicine and Western Medicine Integrated Hospital), 208 Huancheng Dong Road, Hangzhou 310003, Zhejiang Province, People's Republic of China. ${ }^{2}$ School of Life Science and Technology, Xidian University, Xi'an, Shaanxi, China

\section{References}

1. Llovet JM, Ricci S, Mazzaferro V, et al. Sorafenib in advanced hepatocellular carcinoma. The New England journal of medicine. 2008; 359: 378-90.

2. Daher S, Massarwa M, Benson AA, Khoury T. Current and Future Treatment of Hepatocellular Carcinoma: An Updated Comprehensive Review. J Clin Transl Hepatol. 2018; 6: 69-78.

3. van Malenstein H, Dekervel J, Verslype C, et al. Long-term exposure to sorafenib of liver cancer cells induces resistance with epithelial-to-mesenchymal transition, increased invasion and risk of rebound growth. Cancer Lett. 2013; 329: 74-83.

4. Wang S, Cai L, Zhang F, Shang X, Xiao R, Zhou H. Inhibition of EZH2 Attenuates Sorafenib Resistance by Targeting NOTCH1 Activation-Dependent Liver Cancer Stem Cells via NOTCH1-Related MicroRNAs in Hepatocellular Carcinoma. Translational oncology. 2020; 13: 100741.

5. Xin HW, Ambe CM, Hari DM, et al. Label-retaining liver cancer cells are relatively resistant to sorafenib. Gut. 2013; 62: 1777-86.

6. Chai S, Ng KY, Tong M, et al. Octamer 4/microRNA-1246 signaling axis drives Wnt/beta-catenin activation in liver cancer stem cells. Hepatology. 2016; 64: 2062-76.

7. Chen ZZ, Huang L, Wu YH, Zhai WJ, Zhu PP, Gao YF. LncSox4 promotes the self-renewal of liver tumour-initiating cells through Stat3-mediated Sox4 expression. Nat Commun. 2016; 7: 12598.

8. Wang $\mathrm{X}$, Wang $\mathrm{R}$, Bai $\mathrm{S}$, et al. Musashi2 contributes to the maintenance of CD44v6+ liver cancer stem cells via notch1 signaling pathway. J Exp Clin Cancer Res. 2019; 38: 505.

9. Shachaf CM, Kopelman AM, Arvanitis C, et al. MYC inactivation uncovers pluripotent differentiation and tumour dormancy in hepatocellular cancer. Nature. 2004; 431: 1112-7.

10. Toh TB, Lim JJ, Hooi L, Rashid M, Chow EK. Targeting Jak/Stat pathway as a therapeutic strategy against SP/CD44+ tumorigenic cells in Akt/beta-catenin-driven hepatocellular carcinoma. Journal of hepatology. 2020; 72: 104-18.

11. Giannelli G, Koudelkova P, Dituri F, Mikulits W. Role of epithelial to mesenchymal transition in hepatocellular carcinoma. Journal of hepatology. 2016; 65: 798-808. 
12. Mittal V. Epithelial Mesenchymal Transition in Tumor Metastasis. Annu Rev Pathol. 2018; 13: 395412.

13. Shibue T, Weinberg RA. EMT, CSCs, and drug resistance: the mechanistic link and clinical implications. Nat Rev Clin Oncol. 2017; 14: 611-29.

14. Chen X, Lingala S, Khoobyari S, Nolta J, Zern MA, Wu J. Epithelial mesenchymal transition and hedgehog signaling activation are associated with chemoresistance and invasion of hepatoma subpopulations. Journal of hepatology. 2011; 55: 838-45.

15. Mani SA, Guo W, Liao MJ, et al. The epithelial-mesenchymal transition generates cells with properties of stem cells. Cell. 2008; 133: 704-15.

16. Diaz-Lopez A, Diaz-Martin J, Moreno-Bueno G, et al. Zeb1 and Snail1 engage miR-200f transcriptional and epigenetic regulation during EMT. Int J Cancer. 2015; 136: E62-73.

17. Jung HY, Yang J. Unraveling the TWIST between EMT and cancer stemness. Cell Stem Cell. 2015; 16: $1-2$.

18. Lin X, Chai G, Wu Y, et al. RNA m(6)A methylation regulates the epithelial mesenchymal transition of cancer cells and translation of Snail. Nat Commun. 2019; 10: 2065.

19. Serrano-Gomez SJ, Maziveyi M, Alahari SK. Regulation of epithelial-mesenchymal transition through epigenetic and post-translational modifications. Mol Cancer. 2016; 15: 18.

20. Xia H, Ooi LL, Hui KM. MicroRNA-216a/217-induced epithelial-mesenchymal transition targets PTEN and SMAD7 to promote drug resistance and recurrence of liver cancer. Hepatology. 2013; 58: 629-41.

21. Marquardt JU, Factor VM, Thorgeirsson SS. Epigenetic regulation of cancer stem cells in liver cancer: current concepts and clinical implications. Journal of hepatology. 2010; 53: 568-77.

22. Bates SE. Epigenetic Therapies for Cancer. The New England journal of medicine. 2020; 383: 650-63.

23. Fenizia C, Bottino C, Corbetta S, et al. SMYD3 promotes the epithelial-mesenchymal transition in breast cancer. Nucleic Acids Res. 2019; 47: 1278-93.

24. Sarris ME, Moulos P, Haroniti A, Giakountis A, Talianidis I. Smyd3 Is a Transcriptional Potentiator of Multiple Cancer-Promoting Genes and Required for Liver and Colon Cancer Development. Cancer cell. 2016; 29: 354-66.

25. Hamamoto R, Silva FP, Tsuge M, et al. Enhanced SMYD3 expression is essential for the growth of breast cancer cells. Cancer science. 2006; 97: 113-8.

26. Wang S, Zhu Y, He H, et al. Sorafenib suppresses growth and survival of hepatoma cells by accelerating degradation of enhancer of zeste homolog 2. Cancer science. 2013; 104: 750-9. 
27. Tsai CH, Chen YJ, Yu CJ, et al. SMYD3-Mediated H2A.Z.1 Methylation Promotes Cell Cycle and Cancer Proliferation. Cancer research. 2016; 76: 6043-53.

28. Liu $\mathrm{H}, \mathrm{Xu} \mathrm{L,} \mathrm{He} \mathrm{H,} \mathrm{et} \mathrm{al.} \mathrm{Hepatitis} B$ virus $X$ protein promotes hepatoma cell invasion and metastasis by stabilizing Snail protein. Cancer science. 2012; 103: 2072-81.

29. Peserico A, Germani A, Sanese P, et al. A SMYD3 Small-Molecule Inhibitor Impairing Cancer Cell Growth. J Cell Physiol. 2015; 230: 2447-60.

30. Cairo S, Armengol C, De Reynies A, et al. Hepatic stem-like phenotype and interplay of Wnt/betacatenin and Myc signaling in aggressive childhood liver cancer. Cancer cell. 2008; 14: 471-84.

31. Chow EK, Fan LL, Chen X, Bishop JM. Oncogene-specific formation of chemoresistant murine hepatic cancer stem cells. Hepatology. 2012; 56: 1331-41.

32. Liu Y, Deng J, Luo X, et al. Overexpression of SMYD3 was associated with increased STAT3 activation in gastric cancer. Med Oncol. 2015; 32: 404.

33. Mazur PK, Reynoird N, Khatri P, et al. SMYD3 links lysine methylation of MAP3K2 to Ras-driven cancer. Nature. 2014; 510: 283-7.

34. Nagata DE, Ting HA, Cavassani KA, et al. Epigenetic control of Foxp3 by SMYD3 H3K4 histone methyltransferase controls iTreg development and regulates pathogenic T-cell responses during pulmonary viral infection. Mucosal Immunol. 2015; 8: 1131-43.

\section{Figures}




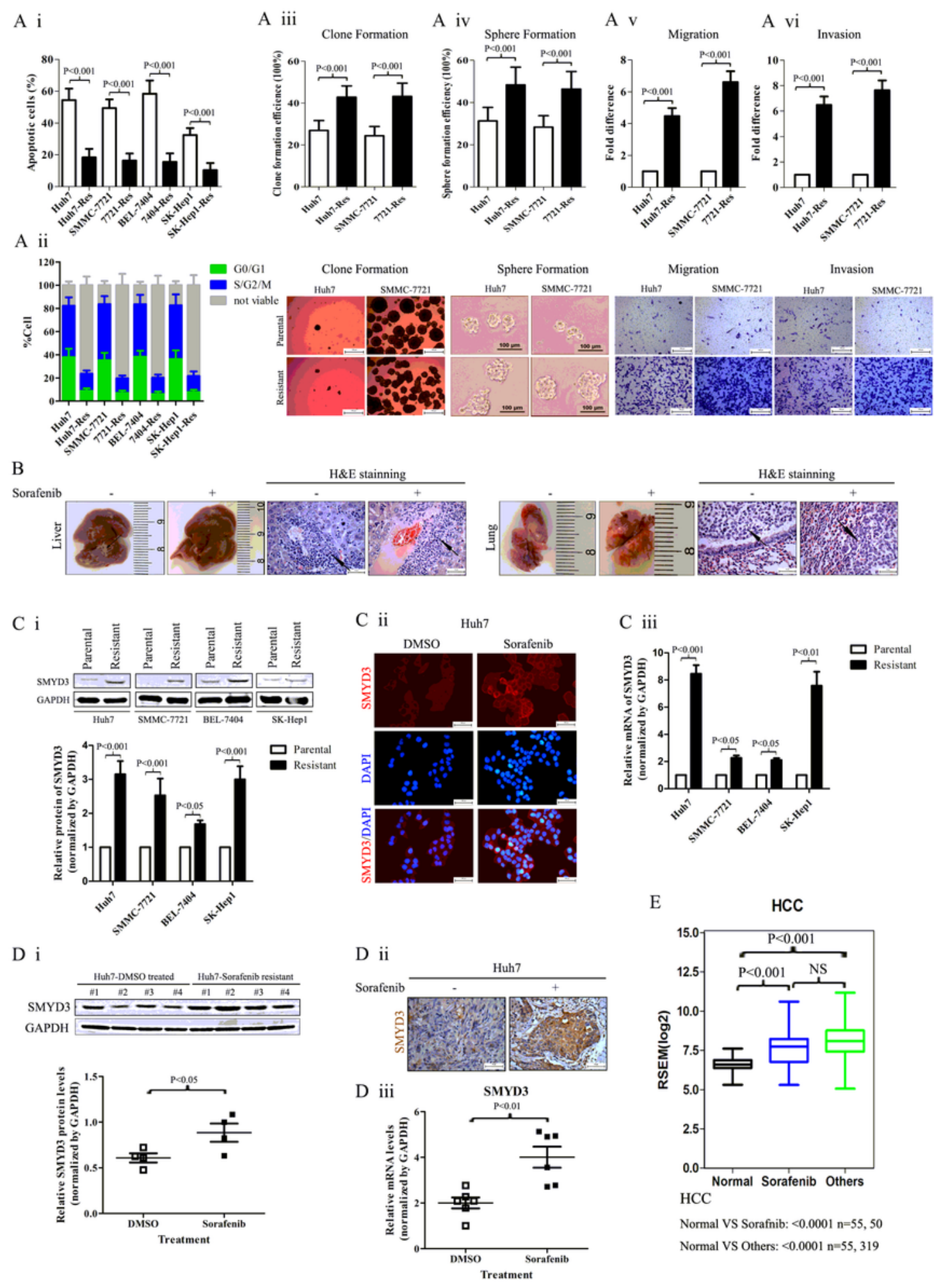

\section{Figure 1}

SMYD3 is overexpressed in sorafenib-resistant tumors and cells. (a) Apoptosis ability (i), cell cycle (ii), tumorigenecity (iii), self-renewal ability (iv), and migration (v) and invasion (vi) ability were checked by Annexin V staining , IP staining, colony and sphere formation assay, and migration and invasion assay in parental and sorafenib-resistant cells. (b) Macroscopic appearance livers and lungs, and representative pictures of livers and lungs with metastatic tumors stained for H\&E. (c) i: Up, immunoblotting analysis of 
SMYD3 and GAPDH in parental and sorafenib-resistant cells. Down, Quantification of relative protein expression of SMYD3/GAPDH is shown. ii: Immunofluorescence staining for SMYD3 expression in parental and sorafenib-resistant cells. iii: Real-time PCR analysis of SMYD3 in parental and sorafenibresistant cells. (d) i: Up, immunoblotting analysis of SMYD3 and GAPDH in DMSO-treated and Sorafenibresistant tumors. Down, Quantification of relative protein expression of SMYD3/GAPDH is shown. ii: IHC staining for SMYD3 expression. iv: Real-time PCR analysis of SMYD3 (down) in DMSO-treated and Sorafenib-resistant tumors. (e) Analysis of SMYD3 expression changes in the human liver cancer samples deposited to "The Cancer Genome Atlas". Patients treated with sorafenib or other drugs. 
A

SoX4

MYC

JAK!
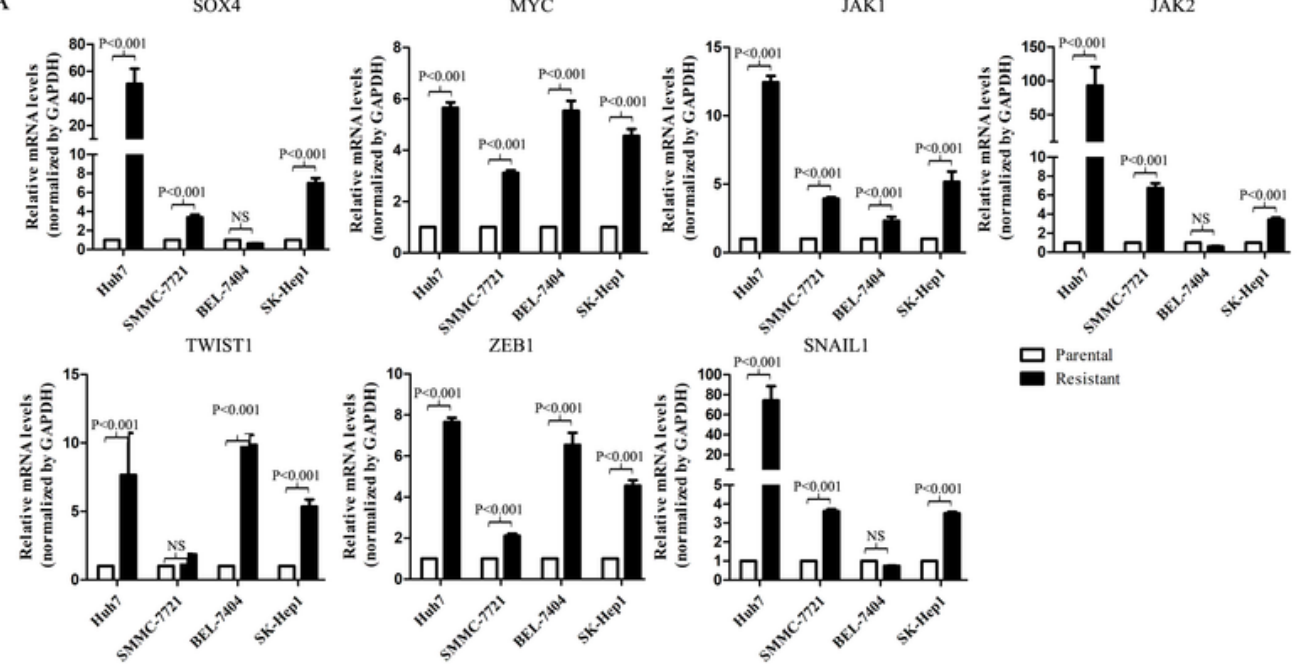

- Resistant

CCNA2

CCNEI
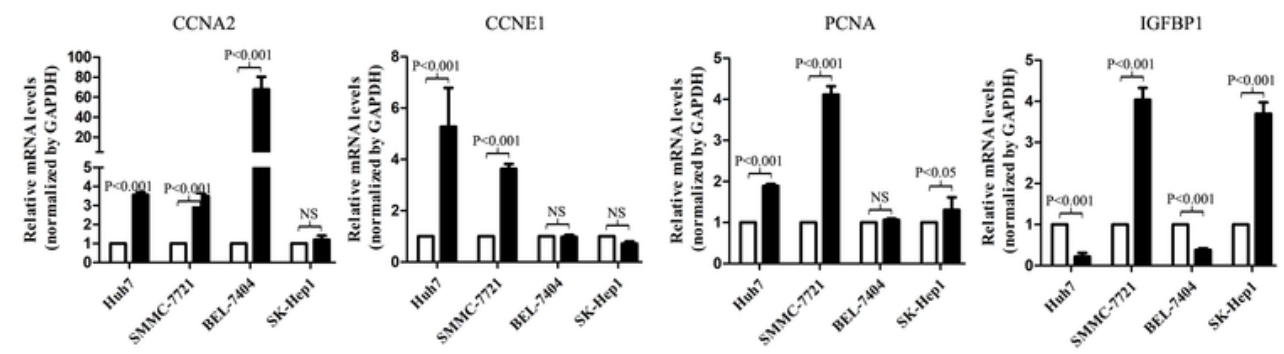

B
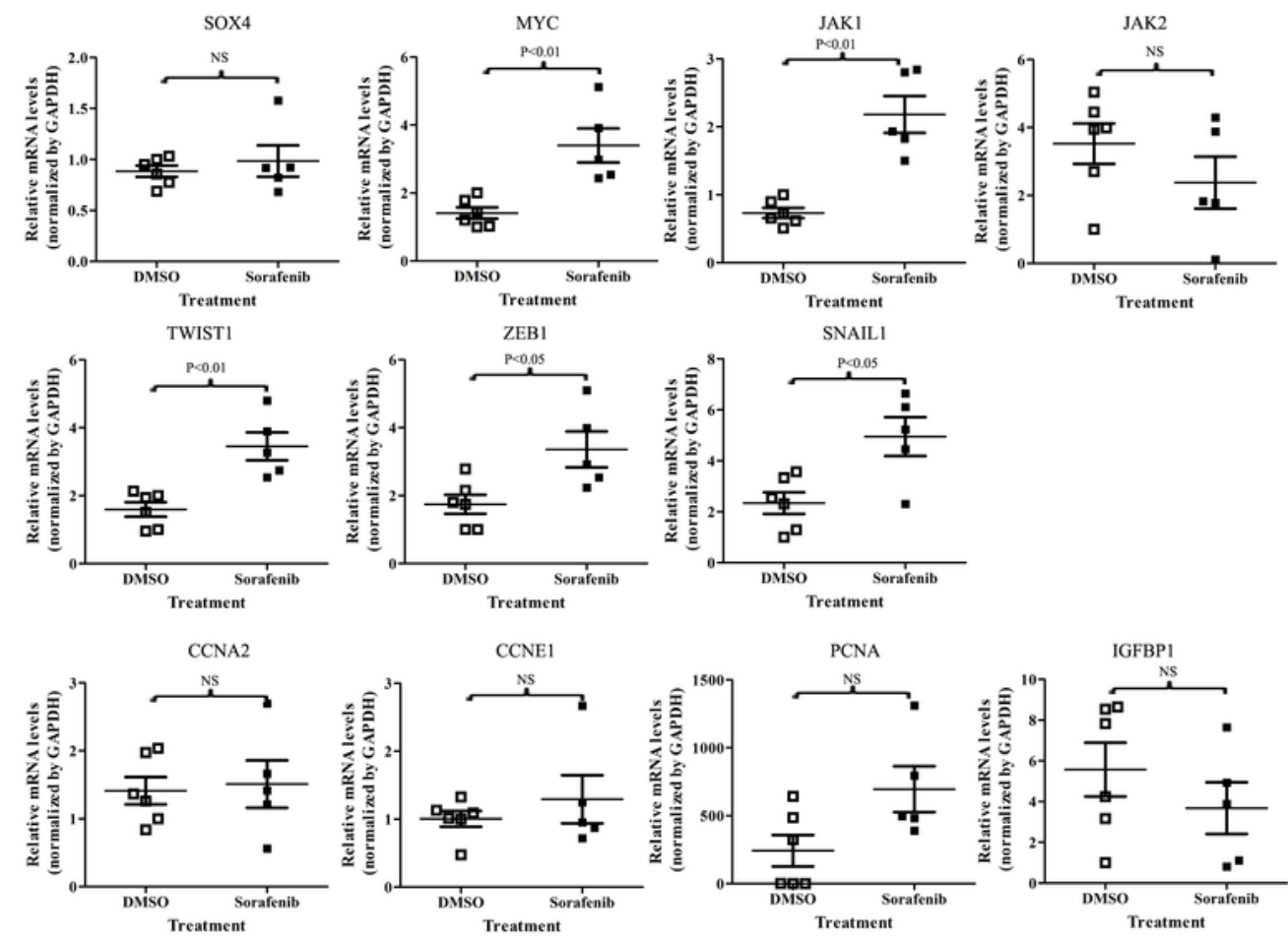

Figure 2

Sorafenib-resistant cells and tumors exhibit widespread up-regulation of multiple cancer-promoting genes. The expression of indicated genes was analyzed in parental and sorafenib-resistant cells (a) and DMSO-treated and Sorafenib-resistant tumors (b) using RT-PCR analysis. mRNA expression for indicated genes relative parental cells or DMSO-treated tumors in indicated HCC cell lines or tumors is shown. Results are expressed as the relative expression (mean $\pm S D$ ). $N=3-6$, each in triplicate; NS, not significant. 
A

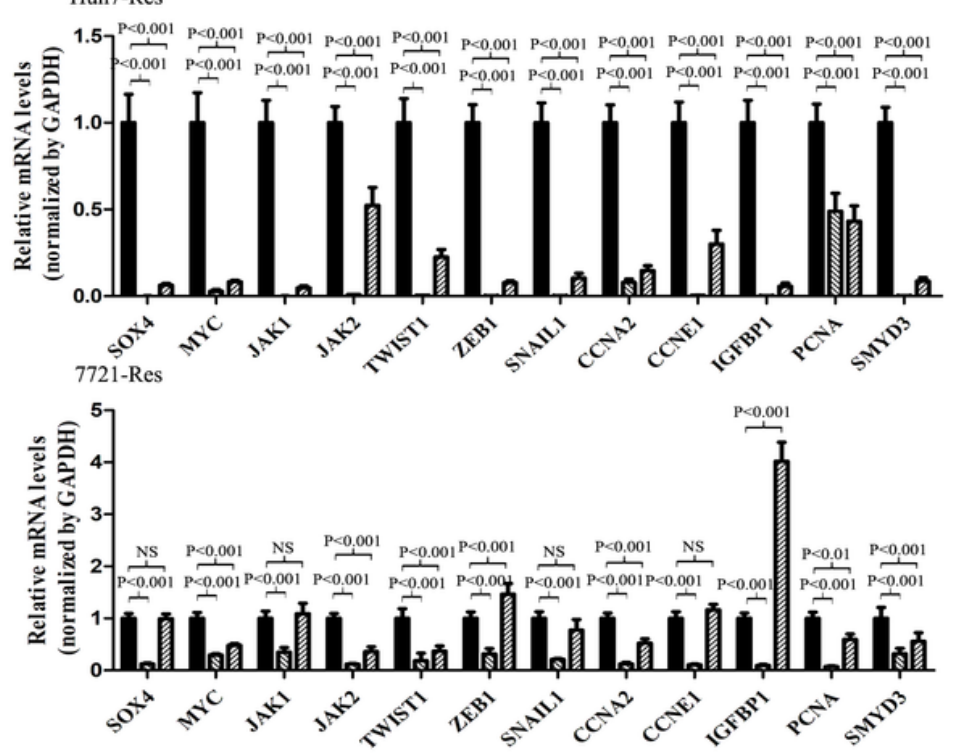

$\mathrm{C}$

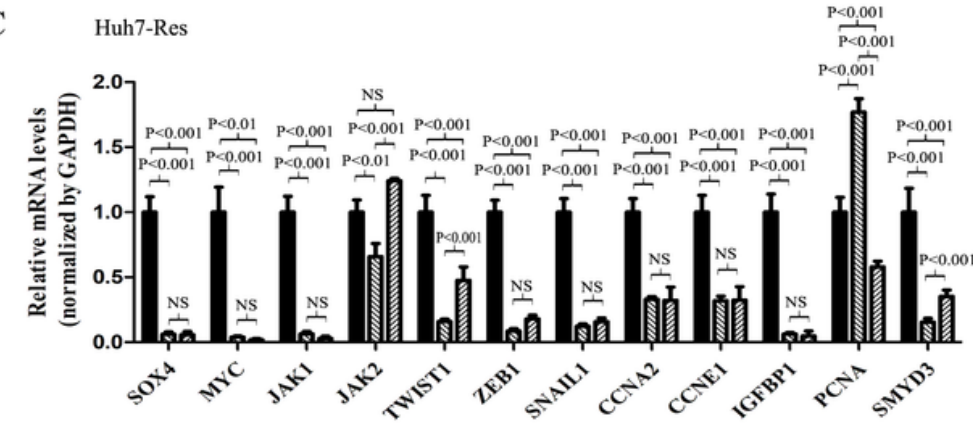

$7721-$ Res

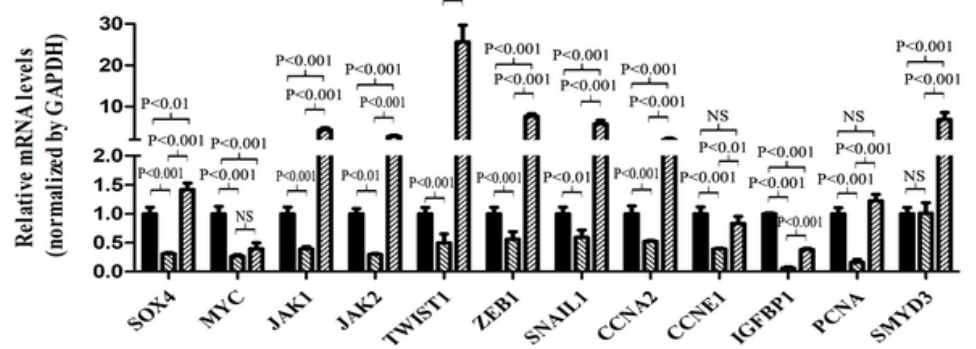

B
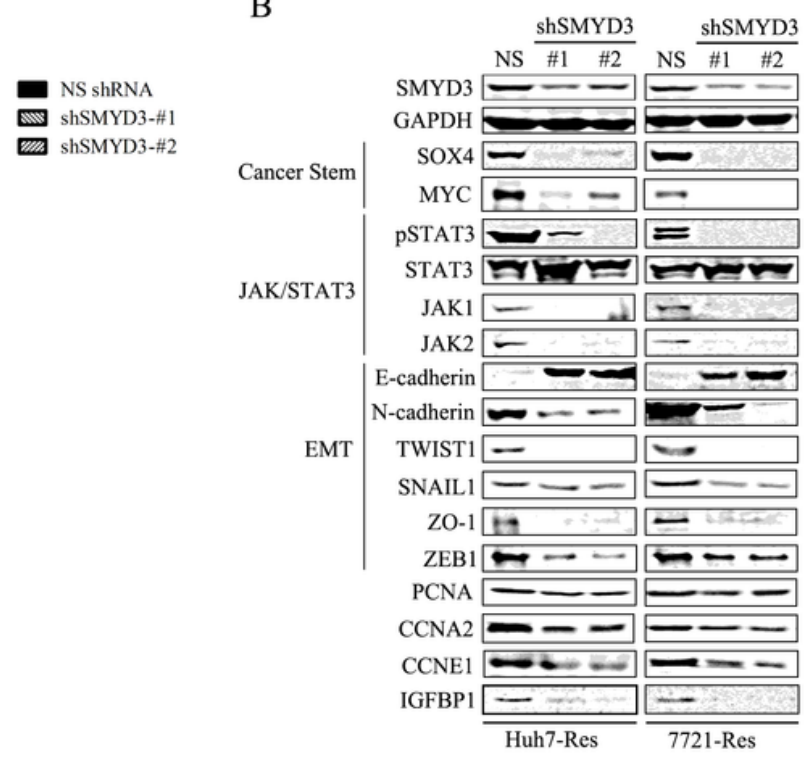

$\mathrm{D}$

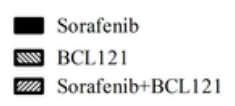

Sorafenib+BCL12

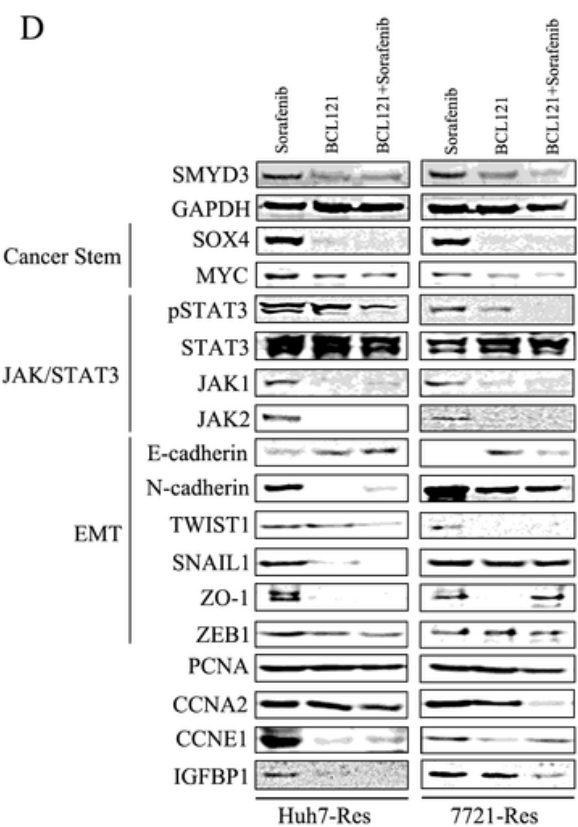

Figure 3

Genetic and pharmacological inhibition of SMYD3 suppresses the expression of multiple cancerpromoting genes and signal pathways in sorafenib-resistant cells. Sorafenib-resistant cells expressed scrambled shRNA (NS shRNA) and two different SMYD3 shRNAs (shSMYD3, \#1 and \#2) (a and b). Sorafenib-resistant cells were treated with SMYD3 inhibitors 10 $\mu$ M BCL121, 10 $\mu$ M Sorafenib, or 10 $\mu$ M Sorafenib+10 $\mu \mathrm{M}$ BCL121, for 48hr ( $c$ and d). Real-time PCR analysis of the indicated genes in parental and sorafenib-resistant cells (a and c, up: Huh7-Res, down: 7721-Res). Immunoblotting analysis of the indicated proteins in parental and sorafenib-resistant cells (b and d, left: Huh7-Res, right: 7721-Res). Results are expressed as the relative expression (mean $\pm S D$ ). $N=3$, each in triplicate. 

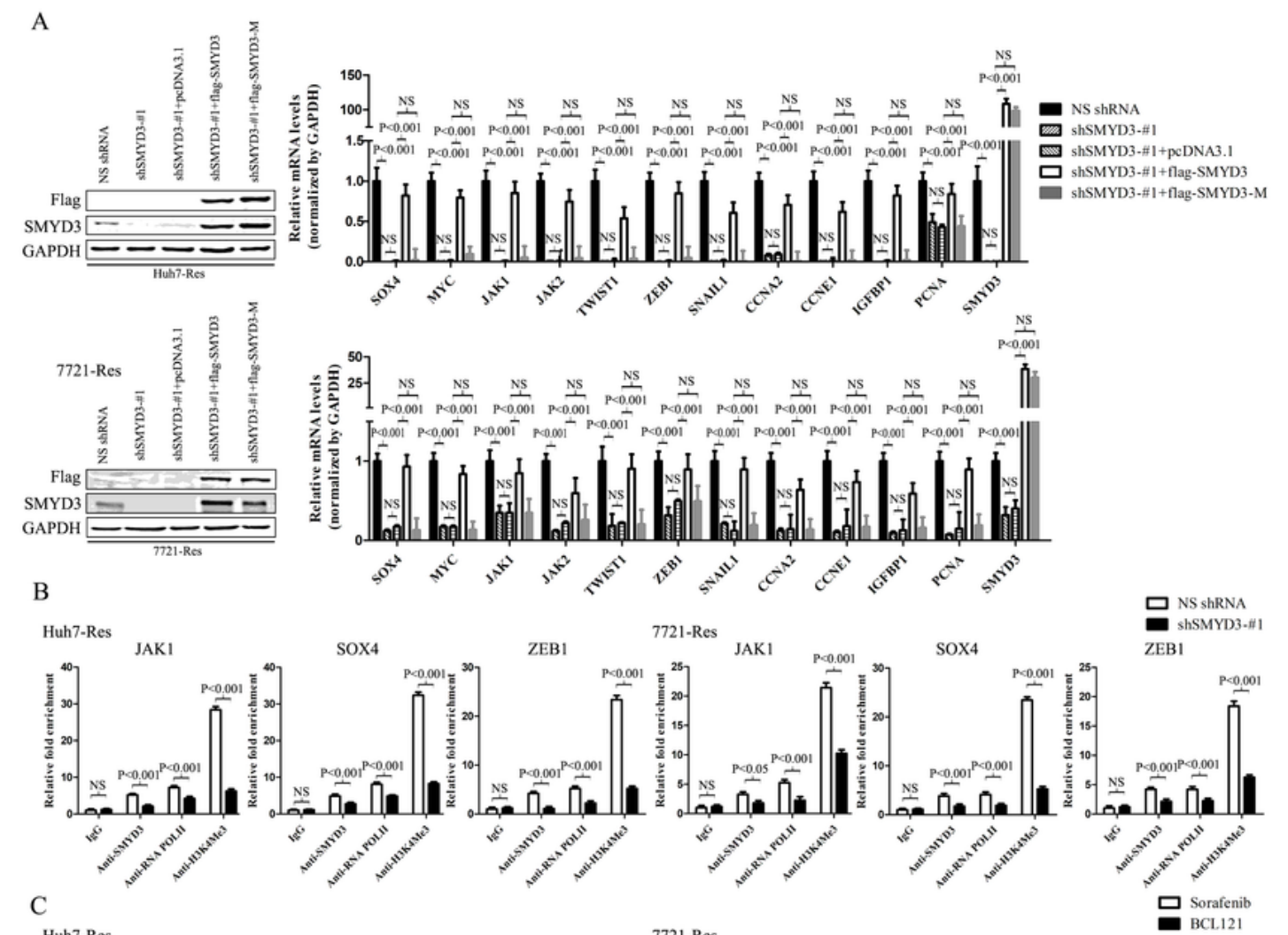

$\mathrm{C}$
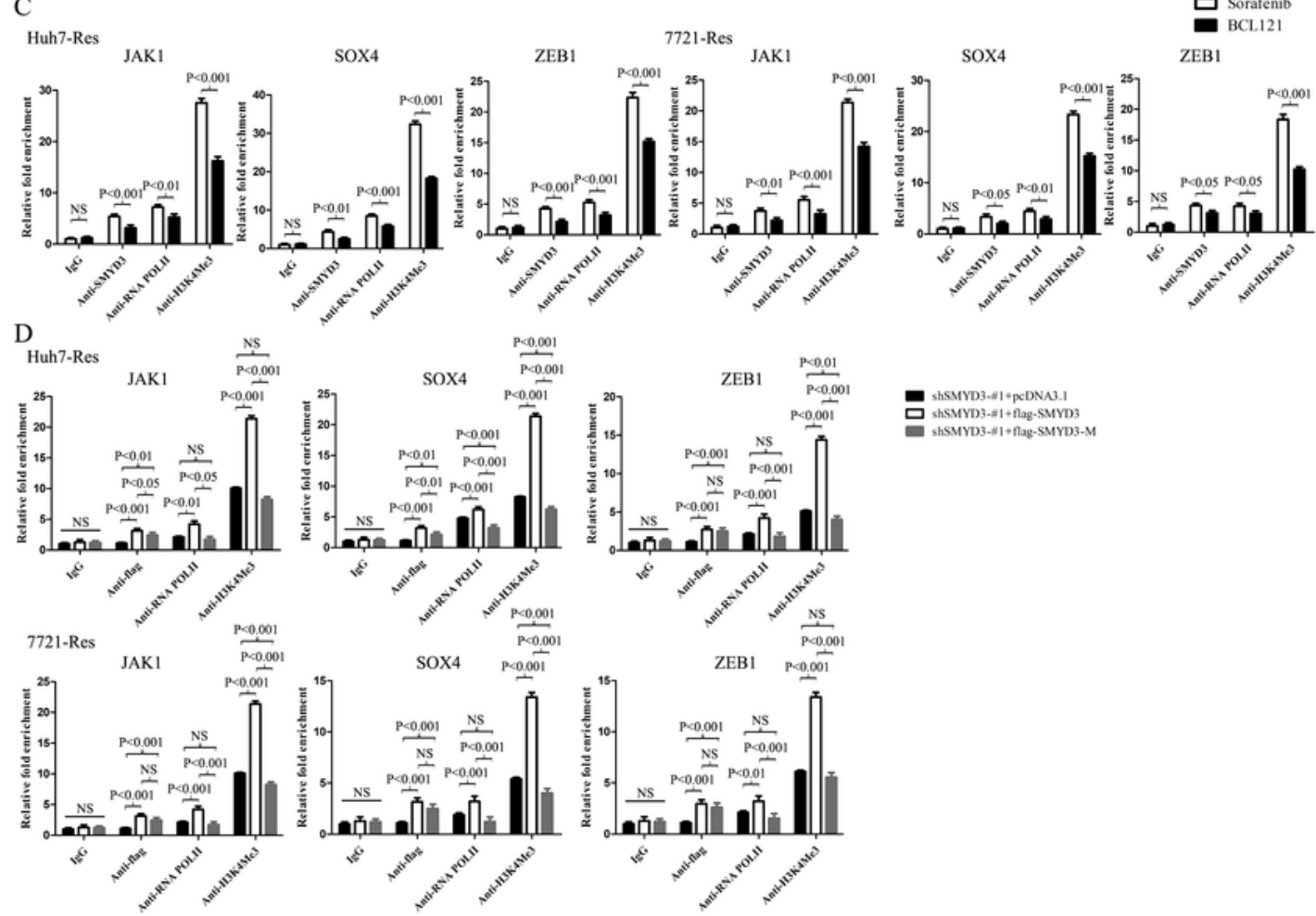

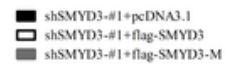
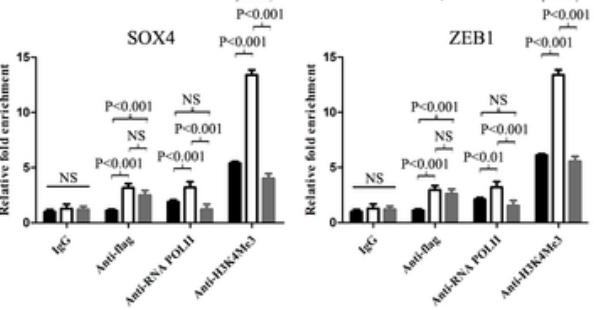

Figure 4

SMYD3 is necessary for the regulation of multiple cancer-promoting genes, dependent on its histone methyl-transferase activity. (a) Effect of overexpressing wide-type SMYD3 (pcDNA3.1-flag-SMYD3widetype, short for "SMYD3") and mutant SMYD3 (pcDNA3.1-flag-SMYD3-Y239F, short for "SMYD3-M") on expression of indicated proteins and genes using immunoblotting analysis (Left) and RT-PCR analysis (Right) in sorafenib-resistant HCC cells with SMYD3 knockdown. (b-c) Effects of the recruitment of 
SMYD3, RNA POLII and the enrichment of H3K4me3 mark on JAK1, SOX4 and ZEB1 loci upon SMYD3 knockdown (b) or SMYD3 inhibition by BCL121(c) using ChIP-qPCR analysis. Sorafenib-resistant cells

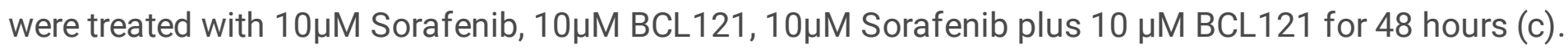
(d) Effects of the recruitment of SMYD3, RNA POLII and the enrichment of H3K4me3 mark on JAK1, SOX4 and ZEB1 gene loci upon SMYD3 or SMYD3-M overexpression in sorafenib-resistant HCC cells with SMYD3 knockdown using ChIP-qPCR analysis. Results are expressed as the relative expression (mean $\pm S D$ ). $\mathrm{N}=3$, each in triplicate; NS, not significant.

A

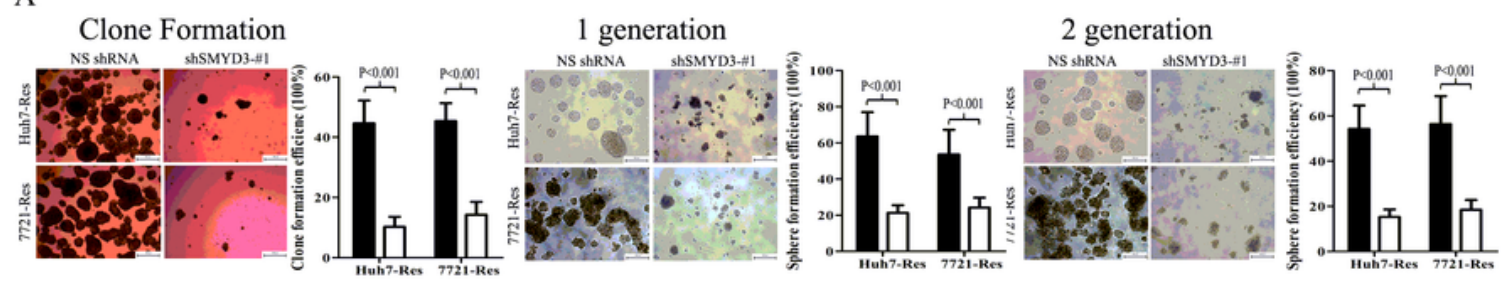

B

\begin{tabular}{|c|c|c|c|c|c|c|}
\hline \multirow{2}{*}{ Cell Dose } & \multicolumn{2}{|c|}{ Huh7-Res } & \multicolumn{4}{|c|}{ 7721-Res } \\
\hline & NS shRNA & shSMYD $3-\# 1$ & & \multicolumn{2}{|c|}{ NS shRNA } & shSMYD $3=1$ \\
\hline $1 \times 10^{2}$ & $2 / 10$ & $0 / 10$ & & \multicolumn{2}{|l|}{$3 / 10$} & $0 / 10$ \\
\hline $1 \times 10^{3}$ & $5 / 10$ & $2 / 10$ & & \multicolumn{2}{|l|}{$4 / 10$} & $2 / 10$ \\
\hline $1 \times 10^{4}$ & $8 / 10$ & $3 / 10$ & & \multicolumn{2}{|l|}{$7 / 10$} & $4 / 10$ \\
\hline \multirow{2}{*}{\multicolumn{3}{|c|}{ Cell Populations }} & \multicolumn{3}{|c|}{ Stem Cell Frequency } & $P$ \\
\hline & & & Lower & \begin{tabular}{|l|l} 
Estimate \\
\end{tabular} & Upper & $P$ \\
\hline \multirow{2}{*}{ Huh7-Res } & \multicolumn{2}{|c|}{ NS shRNA } & 6912 & 3402 & 1675 & \multirow{2}{*}{0.000637} \\
\hline & shSI. & MYD3-\#1 & 46659 & 18733 & 7521 & \\
\hline \multirow{2}{*}{ 7721-Res } & \multicolumn{2}{|c|}{ NS shRNA } & 8720 & 4395 & 2215 & \multirow{2}{*}{0.0129} \\
\hline & \multicolumn{2}{|c|}{ shSMYD3-\#1 } & 33739 & 14622 & 6337 & \\
\hline
\end{tabular}

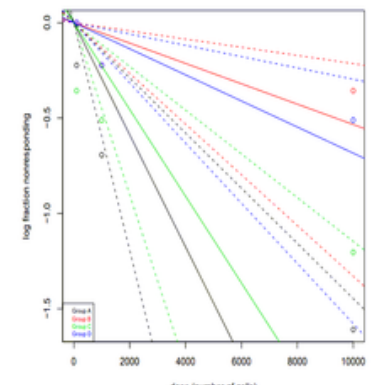

Gruop A: Huh7-Res

Group B: Huh7-Res shSMYD3-\#1 Group C: 7721-Res

Group D: 7721-Res shSMYD3-\#1

C

Clone Formation
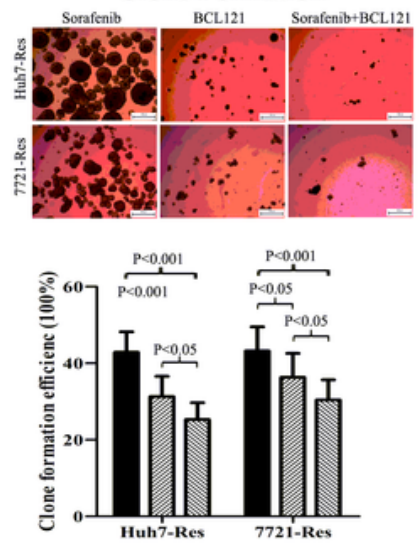

D
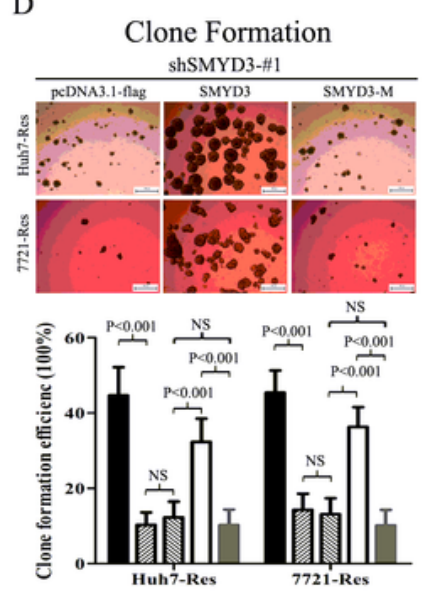
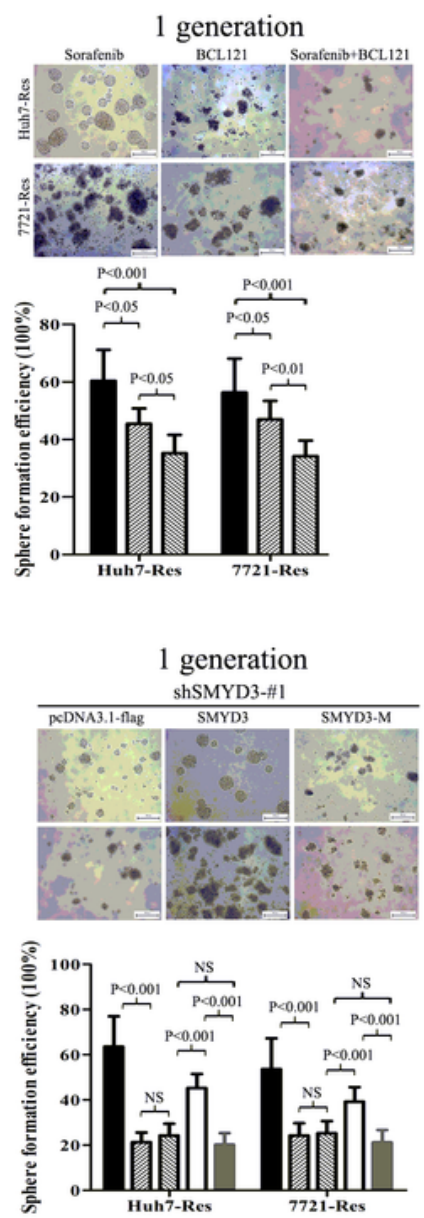
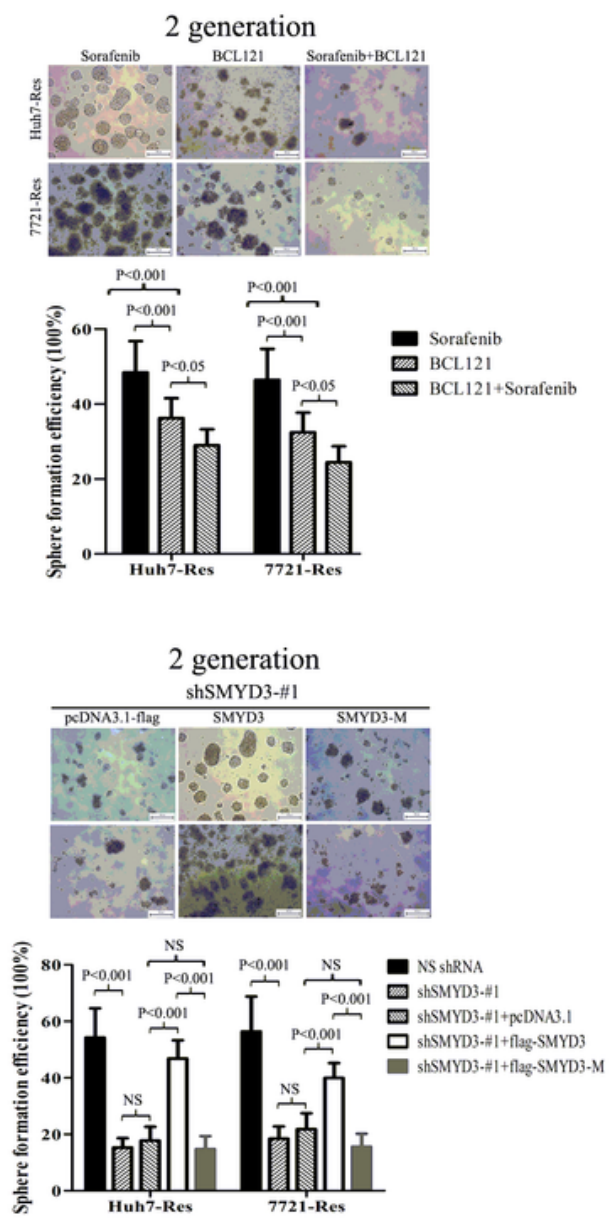


\section{Figure 5}

SMYD3 is essential for the maintenance of liver CSC self-renewal and tumorigenecity in sorafenibresistant cells in vitro. (a) Clone formation efficiency assays and Sphere formation efficiency in the first and second generations, with shSMYD3 or NS shRNA in sorafenib-resistant cells were performed after 2 weeks. (b) Effect of SMYD3 knockdown on the tumor-forming frequency of sorafenib-resistant cells, as determined by limiting dilution assays. CSC frequency was calculated using the ELDA (http://bioinf.wehi.edu.au/software/elda). (c) Effect of SMYD3 inhibitor BCL121 on clone and sphere

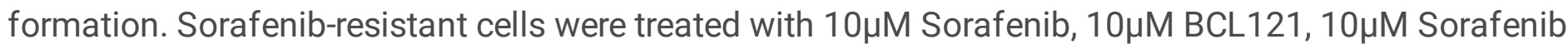
plus 10 MM BCL121. (d) Effect of overexpressing SMYD3 or SMYD3-M on liver CSC self-renewal and tumorigenecity. Sorafenib-resistant cells were stably infected with NS shRNA or shSMYD3-\#1 vectors, and then transfected with SMYD3 or SMYD3-M vector for 24h. Clone formation efficiency assays and sphere formation efficiency in the first and second generations, were performed after 2 weeks. 
A

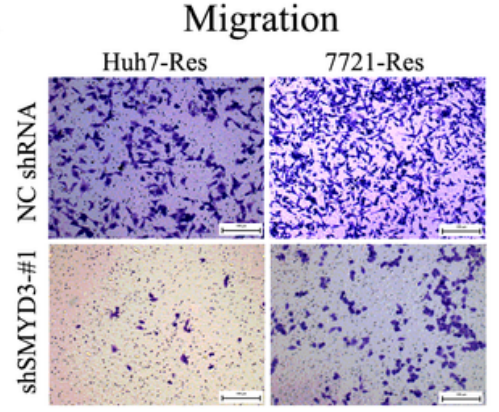

$\mathrm{C}$

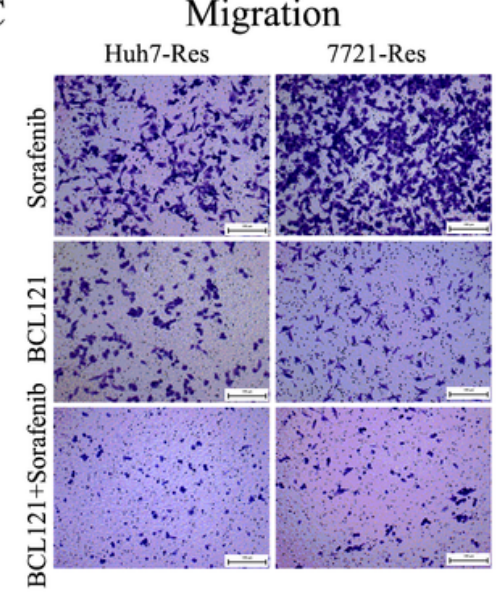

$\mathrm{E}$

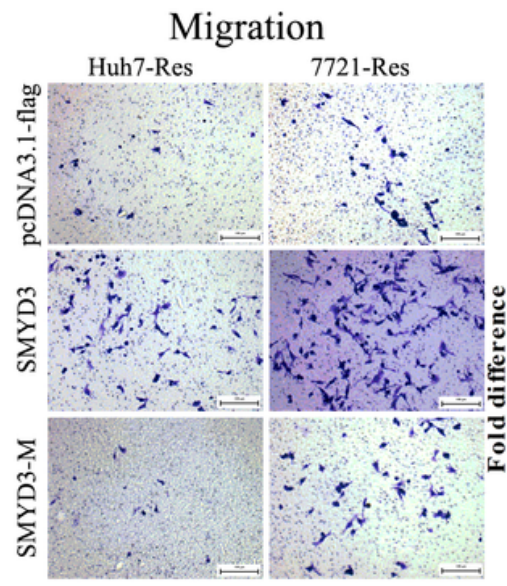

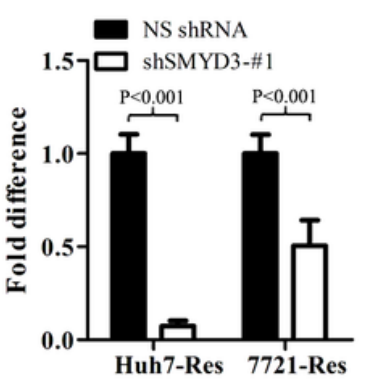

B
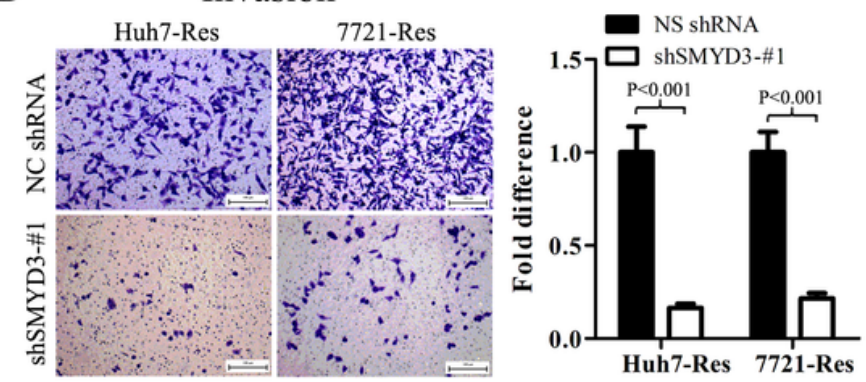

D

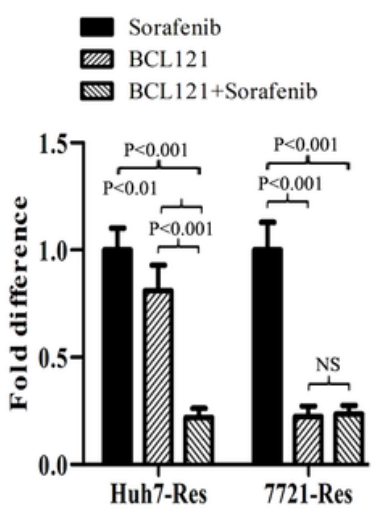

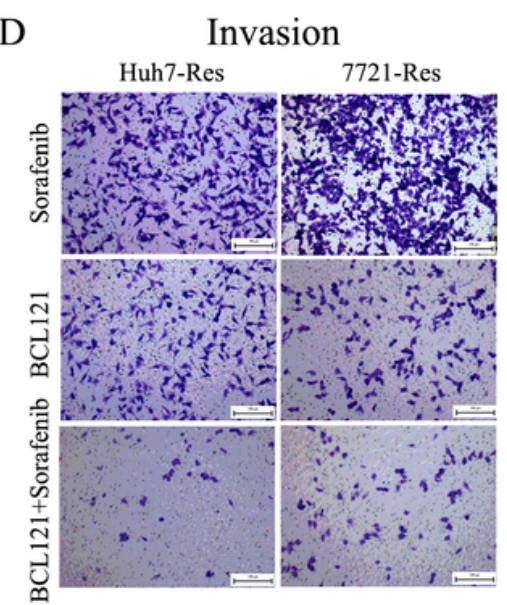

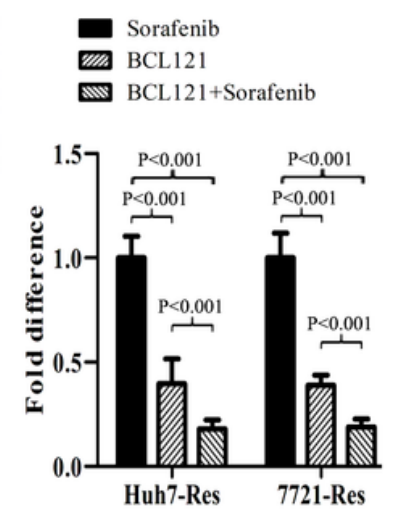

\section{Figure 6}
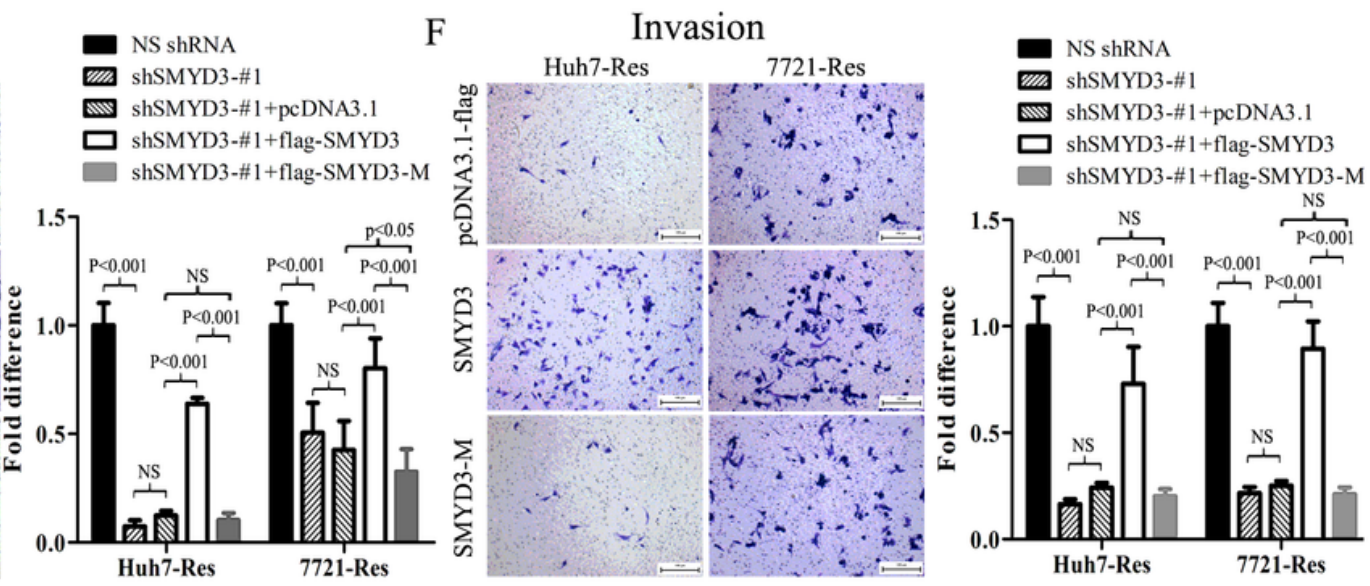

SMYD3 is essential for enhanced migration and invasion abilities in sorafenib-resistant cells in vitro. (a-d) Effects of migration and invasion upon SMYD3 knockdown (a-b) or SMYD3 inhibition by BCL121 (c-d) in sorafenib-resistant cells using migration and invasion assay. Sorafenib-resistant cells were stably

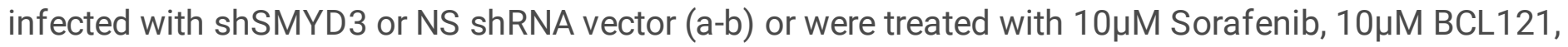

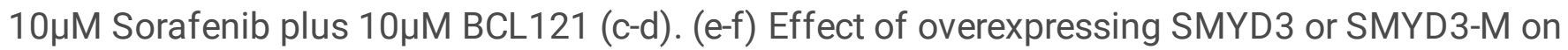
migration and invasion using migration and invasion assay. Sorafenib-resistant cells were stably infected with NS shRNA or shSMYD3-\#1 vectors, and then transfected with SMYD3 or SMYD3-M vector for 24h. 
A

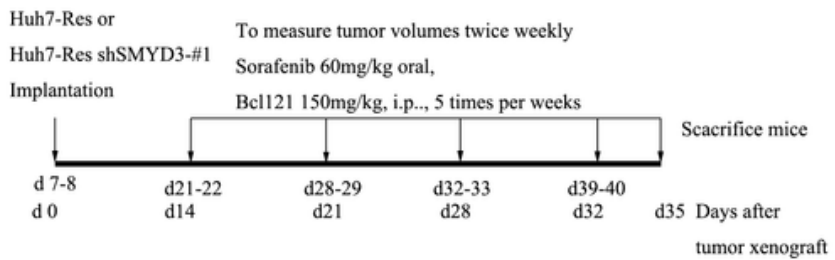

B
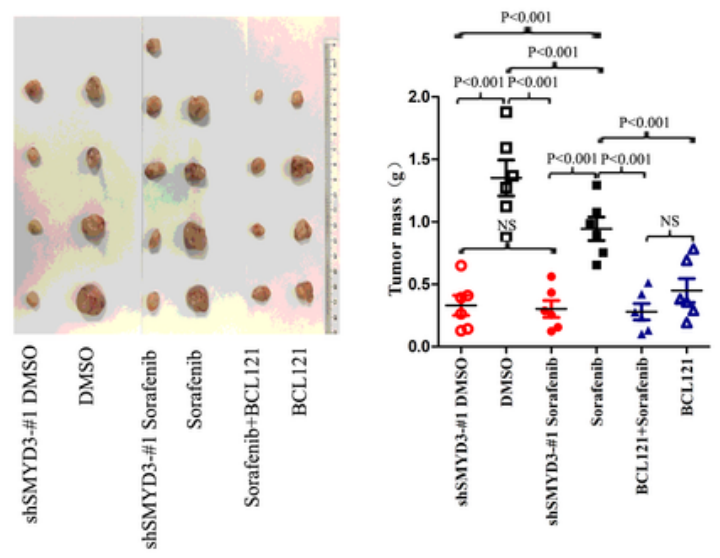

$\mathrm{C}$
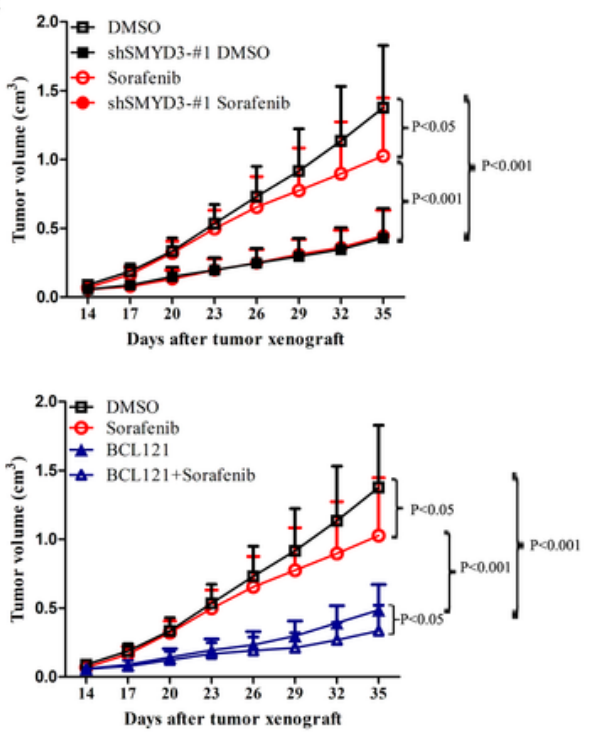

D

H\&E

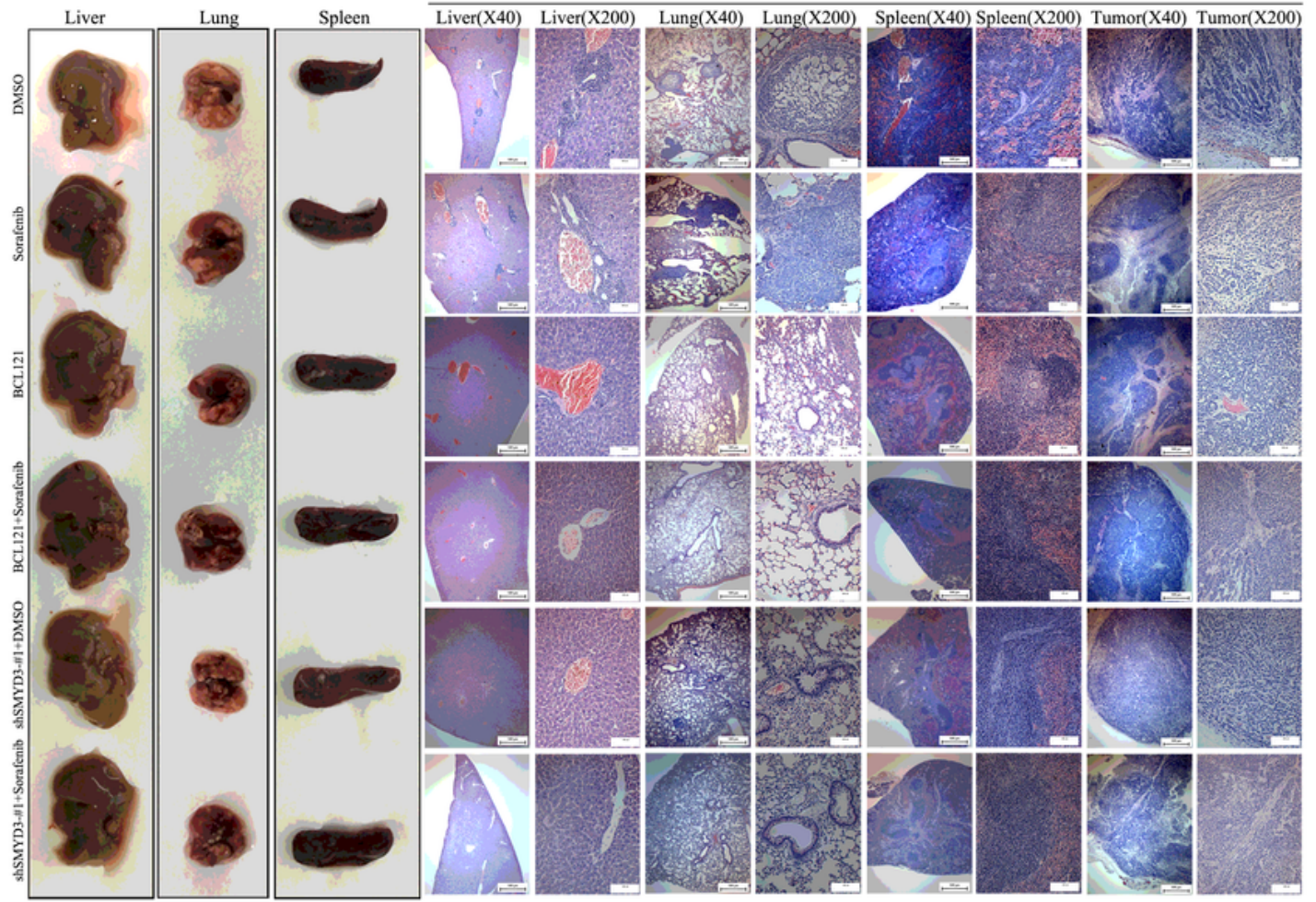

Figure 7

Genetic inhibition and pharmacological of SMYD3 suppresses in vivo tumor growth and liver and lung metastasis. (a) Schematic illustration of treatment schedule. (b-d) Effects of BCL121 and shSMYD3-\#1 on tumor growth of Sorafenib-resistant cells in nude mice; (b) Left: Macroscopic appearance of tumors from indicated treatment. Right: Final tumor masses are represented. (c) Tumor volume was monitored 
every 3 days with indicated (mean $\pm S D, n=4-10 /$ group). (d) Macroscopic appearance of livers, lungs and spleen, and representative pictures of livers, lungs and spleen stained for H\&E. NS, not significant.

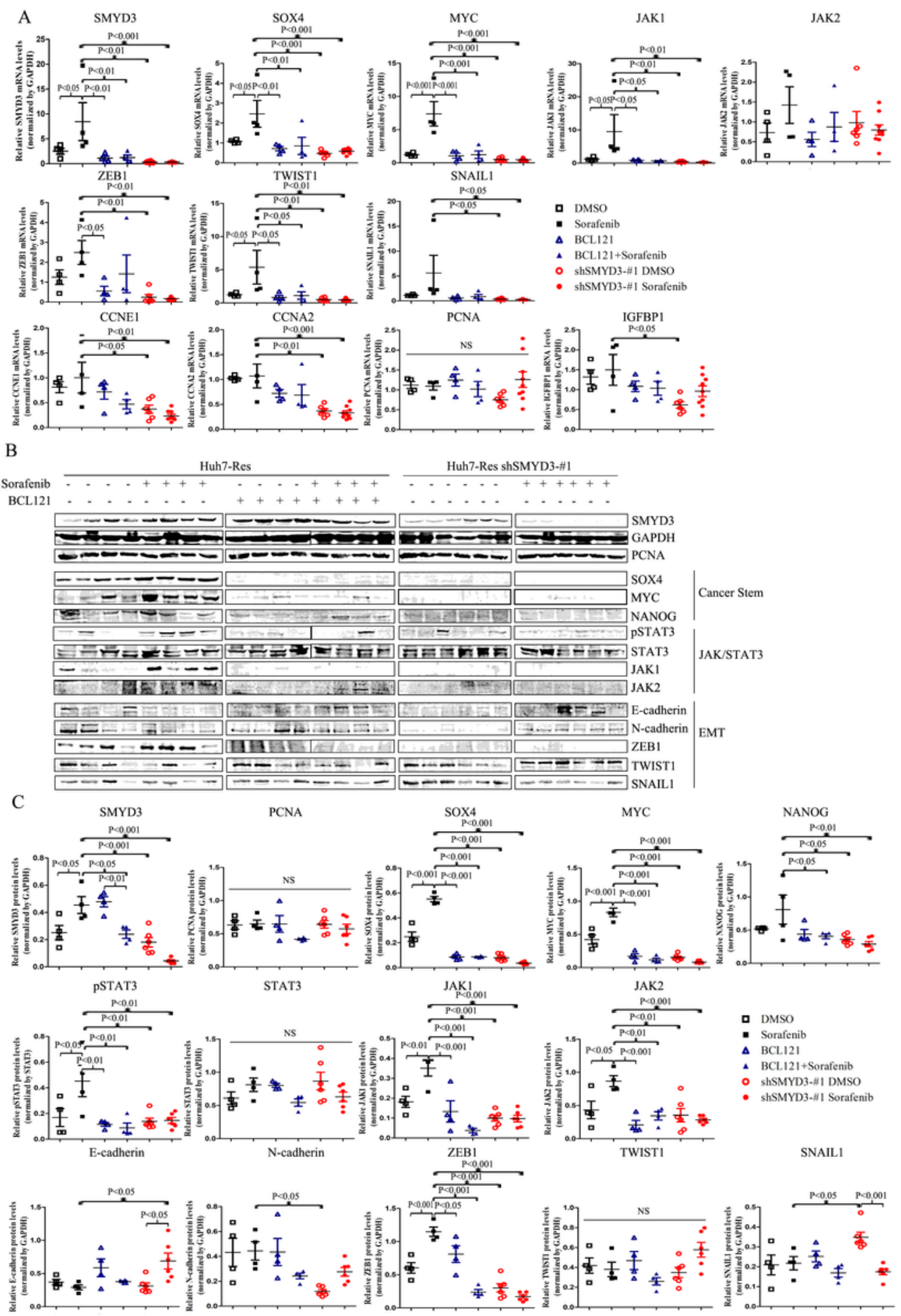

Figure 8

Genetic inhibition and pharmacological of SMYD3 inhibit the expression of multiple cancer-promoting genes and their associated signaling pathway. (a-b) The expression of indicated genes and proteins was analyzed in tumor using qRT-PCR analysis (a) and western blotting analysis (b). (c) Relative protein band 
density of indicated proteins to GAPDH or PSTAT3 to STAT3 by imageJ. Results are expressed as the

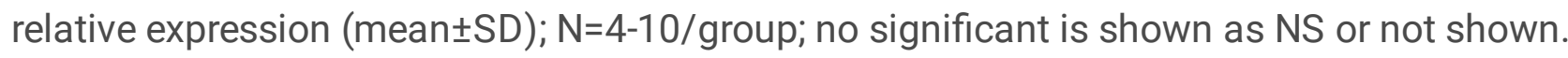

\section{Supplementary Files}

This is a list of supplementary files associated with this preprint. Click to download.

- SMYD3supplemantaldata.xlsx 